\title{
Isospin breaking from diquark clustering
}

\author{
W. R. Gibbs \\ New Mexico State University, Las Cruces, NM 88003
}

\author{
Jean-Pierre Dedonder \\ Sorbonne universités, Université Pierre et Marie Curie, \\ Sorbonne Paris Cité, Université Paris-Diderot et IN2P3-CNRS, UMR 7585, \\ Laboratoire de Physique Nucléaire et Hautes Energies, \\ 4, Place Jussieu, 75252 Paris cedex 05, France \\ (Dated: September 15, 2021)
}

Background Although SU(2) isospin symmetry is generally assumed in the basic theory of the strong interaction, a number of significant violations have been observed in scattering and bound states of nucleons. Many of these violations can be attributed to the electromagnetic interaction but the question of how much of the violation is due to it remains open.

Purpose To establish the connection between diquark clustering in the two-nucleon system and isospin breaking from the Coulomb interaction between the members of diquark pairs.

Method A schematic model based on clustering of quarks in the interior of the confinement region of the twonucleon system is introduced and evaluated. In this model the Coulomb interaction is the source of all isospin breaking. It draws on a picture of the quark density based on the diquark-quark model of hadron structure which has been investigated by a number of groups.

Results The model produces three isospin breaking potentials connecting the unbroken value of the low-energy scattering amplitude to those of the $p p, n n$ and $n p$ singlet channels. A simple test of the potentials in the 3-nucleon energy difference problem yields results in agreement with the known binding energy difference.

Conclusion The illustrative model suggests that the breaking seen in the low-energy NN interaction may be understood in terms of the Coulomb force between members of diquark clusters. It allows the prediction of the charge symmetry breaking interaction and the $n n$ scattering length from the well measured $n p$ singlet scattering length. Values of the $n n$ scattering length around $-18 \mathrm{fm}$ are favored. Since the model is based on the quark picture, it can be easily extended, in the $\mathrm{SU}(3)$ limit, to calculate isospin breaking in the strange sector in the corresponding channels. A natural consequence of isospin breaking from diquark clustering is that the breaking in the strange sector, as measured by the separation energy difference between ${ }_{\Lambda}^{4} \mathrm{H}$ and ${ }_{\Lambda}^{4} \mathrm{He}$, is several times larger than that seen in the comparison of three-nucleon mirror nuclei as observed experimentally.

\section{INTRODUCTION}

In spite of the very significant progress that has been made in the study of Quantum Chromodynamics (QCD), the understanding of the low-energy nucleon-nucleon (NN) interaction in terms of parton degrees of freedom remains elusive. The short-range part of the potential has been calculated with parton degrees of freedom in a number of cases, often with one gluon exchange (which also involves two quark exchange) in first order perturbation theory. Such theories tend to give no (or little) attraction. Since the twopion exchange produces a strong intermediate-range attraction [1] it might be thought that this mechanism alone would suffice. An attempt [2] to marry the short range quark contribution [3 5] to the well known one- and two-pion exchange did not succeed. While empirical evidence of two-pion exchange exists [6] it appears that it does not provide all of the intermediate range potential strength needed.

When the confinement volumes of two nucleons begin to overlap, direct quark-quark interactions can occur through non-color-singlet exchanges producing quark clustering among quarks originating in different nucleons. Underlying this picture is the notion that the NN interaction involves sharing of quarks between the two incident particles when they overlap, thus contributing to the NN potential. Even if this clustering occurs in an isospin symmetric manner (which we assume), the different charges of the quarks will 
produce energy differences which will break the $\mathrm{SU}(2)$ symmetry. It is this source of isospin breaking that we treat in this paper. Since the Coulomb interaction is hidden in the correlations, we refer to the effect as "crypto-Coulomb" (CC). We treat only the valence quarks even though the sea quarks would be expected to participate as well in a more realistic calculation.

In a diquark-quark model of the nucleon the third (unpaired) quark in each nucleon can combine in the overlapping and enlarged confinement region thus adding to the intermediate range attraction. Models in which quark-quark interactions have been included in a single baryon have been moderately successful [7-9] in predicting the mass spectrum.

Here we focus on a more modest goal than producing a full NN potential; instead, we look at the result of this type of interaction and study the Coulombic effect of quark clustering in the valence quark density and compare the result with experimentally observed isospin breaking to infer the parameters describing the quark density.

Not having available the strong potential which would be the result of such a stronginteraction model, we use a phenomenological potential of the Reid soft-core type [10] which has a short-range repulsion, medium range attraction and one-pion-exchange (OPE) tail to provide the basic $\mathrm{SU}(2)$ invariant interaction. This potential is determined by fitting $p p$ scattering data below $30 \mathrm{MeV}$ and then used to calculate the $n n$ and $n p$ effective range parameters and compare them with data. The entire process uses a unique quark density (same for $n n, p p$ and singlet $n p$ pairs) with the isospin breaking being produced solely by the charge differences between the valence quarks themselves.

Section II reviews the evidence for diquark clustering in the interior of hadrons along with a discussion of the expected role of diquark clustering in low-energy NN scattering. In Section III a brief summary of the data on isospin breaking in the NN interaction and the status of the theory are reviewed. In Section IV a simple model is introduced which is based on the naïve quark model but satisfies many of the conditions just discussed. In section $\mathrm{V}$ the method used for extracting the strong phase shifts from the data is given. Section VI gives the results of the model. An extension of these ideas to the strange sector $(\Lambda-N)$ is presented in Section VII. Section VIII gives a short discussion with conclusions.

\section{DIQUARK MODELS}

While diquark models have been around for some time, there has been an increase in interest recently due to new experimental results. In this section we review briefly this history.

The model of nucleon as a diquark (D) quark (q) pair is as old as the idea of quarks themselves. When Gell-mann introduced the name "quark" he discussed the possible existence of the diquark at the same time [11]. Ida and Kobayashi [12], and Lichtenberg and Tassie [13] were the first to consider the diquark-quark model. Ono [14] and Lichtenberg et al. [15, 16] studied the mass spectrum which would be generated by such a model. Abbott et al. [17] saw evidence for a spin-one diquark in the need for an explanation of the large ratio of longitudinal to transverse photon absorption. Gunion [18] saw the need

for a diquark in the form of the structure functions of the nucleon. Later Mineo et al. [19] calculated the structure functions with a diquark model.

The existence of diquark correlations in the nucleon is claimed to be supported by a large body of experimental evidence (see ref. [20] for a summary of the situation in 1993). Lattice gauge theory has provided some theoretical support [21 24] although the size of the diquark extracted is often larger than that assumed by typical models, but not 
always [25].

Much remains to be known about the detailed properties of these correlations. There seem to be indications for both a spin-zero and a spin-one form. The spatial extent of the correlations also varies with the specific model treated. A typical result from the lattice is that the spatial size of the axial vector diquark is larger than that of the scalar diquark. Weiss et al. [26], in a Nambu-Jona-Lasinio model, found that a larger axial vector diquark was necessary in order to have the square charge radius of the neutron be negative. See also Ishii et al. [27] for a similar model.

One diquark model of the nucleon which is particularly successful is the one due to Keiner [28]. While many diquark-quark calculations do not respect isospin invariance, Keiner presented a diquark model for the nucleon which gives an excellent fit to the electromagnetic properties of the nucleon and does respect $\mathrm{SU}(2)$ symmetry. In order to conserve isospin he included both scalar and axial vector diquarks of the same mass and size $(0.24 \mathrm{fm})$.

More modern treatments [29, 30] deal with heavy baryons. Cloët, Bentz and Thomas [31] use diquarks to calculate clustering effects on the nucleon elastic form factors. Santopinto and Ferretti [32] treat strange and non-strange baryons. Another model which leads to quark-quark correlations is the Flux-tube model [8, 9].

More recently, a diquark-antidiquark structure has been studied [33 50] in the form of $[Q q][\bar{Q} \bar{q}]$ for recently observed particles by the BaBar [51], Belle [52], and LHCb [53] collaborations.

Of perhaps more relevance for $\mathrm{NN}$ scattering are the nearly degenerate light scalar mesons $\left[f_{0}(980)\right.$ and $\left.a_{0}(980)\right]$ which have long been a problem to understand in a $q \bar{q}$ model. They have often been viewed as meson-meson molecular states but more recently the interest has increased to study them in the diquark-antidiquark picture [54 60]. In summary, it seems that the idea of diquark clustering in the internal structure of hadrons, light or heavy, is widespread and has many supporters.

Diquark models would seem to have taken a blow when it became evident that the valence quarks carry only a fraction of the spin of the proton. However, Myhrer and Thomas [61] and Thomas [62] pointed out that relativistic effects and the pion cloud could explain this apparent lack. Shortly thereafter, Cloët and Miller published [63] a diquark-quark model of the nucleon in which the axial vector diquark plays an essential role by contributing negatively to the spin sum thus reducing the apparent fraction of the spin carried by the quarks. In their fit to the electromagnetic form factors they found the axial vector diquark to have a smaller mass than the scalar diquark, contrary to most other models, a point that remains to be understood.

Although we know of no current theory which takes into account the clustering of pairs of quarks into diquarks for the $\mathrm{NN}$ interaction in free space, there have been treatments of the nucleonic interaction in nuclear matter in Landau-Migdal models [64, 65]. There is also a study of the transition of nuclear matter to quark matter where a condensate of diquarks is needed to obtain the phase change [66]. The rearrangement of quarks into quark pairs has also been treated in the meson-meson interaction [67].

It is hard to imagine that quark clustering will not play some role in the short-to-midrange $N N$ interaction. When the two confinement volumes begin to overlap (when they touch) the quarks will be free to move about in the aggregated volume so that the two (initially) unpaired quarks can combine to form a diquark thus lowering the total mass of the two-nucleon system thereby creating an attractive potential. This picture is especially favorable at very low energies where the overlap of the two systems exists for a long time allowing the quarks ample opportunity to rearrange themselves. 
The construction of such a theory is well beyond the scope of this paper. Instead, we look at the connection between the clustering which might be generated by such a theory and isospin breaking observed in the NN system in the singlet scattering lengths.

\section{OVERVIEW OF ISOSPIN BREAKING IN THE NN INTERACTION}

In 1974 it was suggested [68] that it might be possible to learn about the short-range $\mathrm{NN}$ interaction from the study of isospin breaking in this interaction. It is the purpose of this paper to present a schematic model realizing this idea using as the only source of breaking the Coulomb interaction between quark pairs.

$\mathrm{SU}(2)$ symmetry (isospin) is one of the fundamental building blocks of QCD. It is known that this symmetry is broken by the Coulomb interaction but it is not known to what extent it is broken in the strong interaction.

One of the most obvious manifestations of isospin breaking is the difference in the nucleon masses. It is generally believed that the neutron-proton $(n p)$ mass difference is due to the up-down quark mass differences [69-72]. While the ud quark mass difference is of the same order as the interior Coulomb effects we will treat, it will not enter into our considerations for the following reason: while the different singlet nucleon pairs will indeed have different total masses, they will not depend on the distance between the centers of mass of the nucleons so that there is no localized potential to use for the calculation of the scattering or binding of nucleons. The result is constant energy shifts which are expressed as the difference in nucleon masses. The nucleon masses will affect the kinetic energy contribution to the bound states and the phase space of the scattering states leading to a small breaking that can be treated in the hadronic calculations needed to remove the conventional Coulomb effects.

Other examples of isospin breaking exist in nuclear physics and it is important to know if they come from additional fundamental breaking or if they can be explained by the electromagnetic interaction. A special case of the breaking is a failure of charge symmetry (CSB) wherein the $n n$ interaction is different from the "Coulomb corrected" $p p$ interaction. A classification of the types of isospin breaking was given by Ref. [73]. One review of the breaking as of 1990 was given in Ref. [74], another in 2006 [75] and again in 2009 [76].

By making the exchange neutron $\Leftrightarrow$ proton in a nuclear system, i.e., by comparing the energies of mirror nuclei, a measure of the CSB can be obtained. In order to carry out this program the ordinary Coulomb interaction must be taken into account. A great deal of work has been done in this direction.

\section{A. The Okamoto-Nolen-Schiffer anomaly}

Discussions of the CSB problem often start with the Okamoto-Nolen-Schiffer anomaly [77, 78] which is the observation that the addition of the conventional Coulomb force between protons does not give enough energy difference between members of a nuclear isospin doublet. By the conventional Coulomb force we mean a $e^{2} / r$ potential at large distances with a continuation for smaller $r$ representing some sort of nucleon charge density, often taken as a uniformly charged sphere. We denote this potential by $V_{C}(r)$. The same discrepancy is observed in the binding energy difference between ${ }^{3} \mathrm{He}$ and ${ }^{3} \mathrm{H}$ (see next section). 


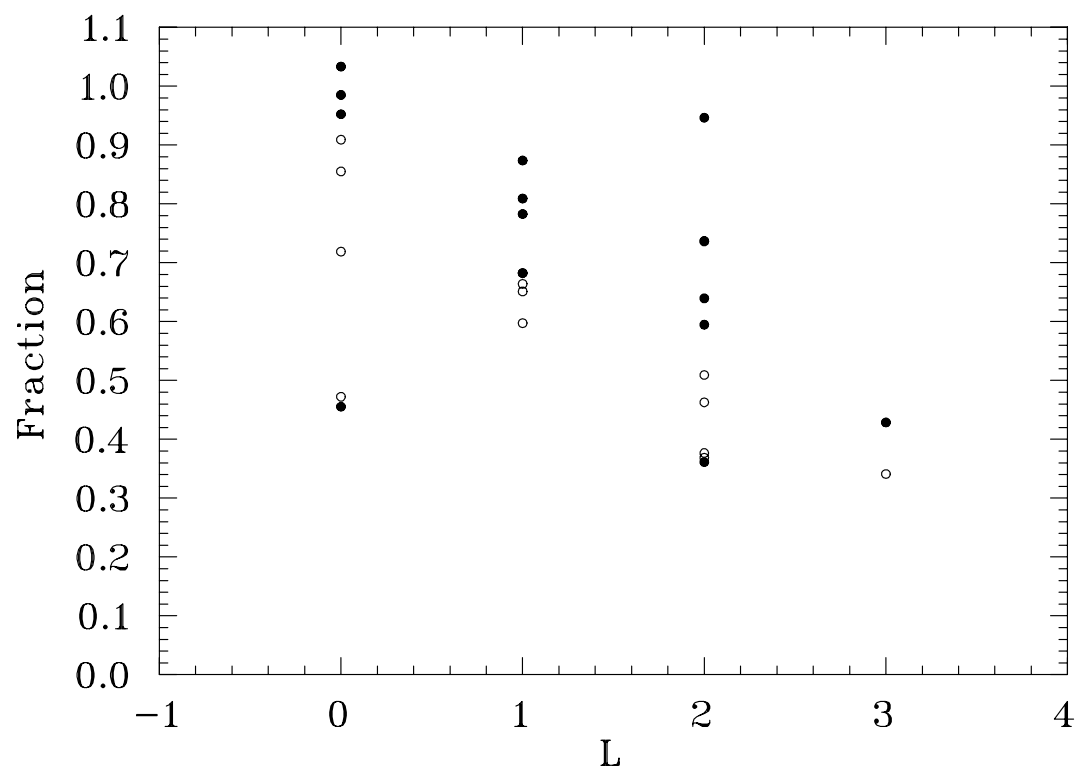

FIG. 1: The dots represent the ratio of the calculation of Blunden and Iqbal [79] based on the $\rho-\omega$ and $\pi-\eta$ charge symmetry breaking potentials of [80] to the CSB energies of Sato [81] as a function of the angular momentum of the shell. The solid dots correspond to the SKII results [82], the open circles to DME calculations [83].

In an attempt to explain this discrepancy by a difference in the fundamental interaction the exchange of heavy mesons (primarily the mixing of the $\rho$ and $\omega$ mesons) [80] has been proposed [79]. These mesonic CSB potentials [80] give the right order of magnitude [79] but they appear to fail quantitatively for the higher angular momentum shells as can be seen in Fig. 1 where the fraction of the needed additional energy provided is shown. The discrepancy in the prediction seems to increase for the larger systems.

Sato 81], who did the calculation of the Coulomb energy from the nuclear wave functions from which the inadequacy of the pure ordinary Coulomb force is obtained, attempted to fit the missing energies with an arbitrary charge-symmetry-breaking potential constrained only to have a range, less than or equal to, one pion exchange but did not find a fit. The calculations of Blunden and Iqbal [79] were done with shell-model wave functions and hence did not have a short-range repulsive interaction excluding the NN wave function from short relative distances. Since the mesonic CSB potentials [80] have a very short range (essentially the same as the one-boson-exchange often used to provide the source of the strongly repulsive short-range potential), one might question the validity of this procedure since it might be expected to overestimate the effect.

\section{B. Three-body comparisons}

While the mirror nuclei comparison offers several useful features, in particular the possibility to change the range over which the breaking interaction is being sampled by varying the atomic number, there is a complication in that a reliable structure calculation is needed. As seen from Fig. 1 considerable variation is present from the difference in the two types of shell-model calculations [82, 83] considered.

The comparison of the binding energy of ${ }^{3} \mathrm{He}$ and ${ }^{3} \mathrm{H}$ offers a way of examining charge symmetry breaking in which the uncertainties due to nuclear structure are largely absent. An additional advantage is that the short-range repulsion of the unbroken NN interaction can be taken into account by the use of "realistic" potentials. The conventional Coulomb 


\begin{tabular}{|c|c|c|}
\hline$a_{N N}(\mathrm{fm})$ & Reaction & Reference \\
\hline$-23.748 \pm 0.009$ & np scattering from ortho- and para-hydrogen & {$[86]-[88]$} \\
\hline$-17.3 \pm 0.4$ & Coulomb corrected pp scattering & {$[74,75,89]$} \\
\hline$-18.5 \pm 0.3$ & nn final state interaction in $\pi^{-} d \rightarrow n n \gamma$ and nd break-up & {$[90]-[95],[96]$ and $[100]-[102]$} \\
\hline$-16.1 \sim-16.3$ & nn final state interaction in nd break-up & {$[74,75][97,98]$} \\
\hline
\end{tabular}

TABLE I: Experimental determinations of the singlet NN scattering length

force can be included in Fadeev calculations to any desired accuracy.

In the three-nucleon system it is found that the conventional Coulomb potential supplies about $85 \%$ of the difference in binding energy (648 keV of the 764 observed) and the remaining $15 \%$ can be reliably attributed to CSB beyond what has been put in to the calculation so far. Wu, Ishikawa and Sasakawa [84] considered a number of small effects (including magnetic interactions and the kinetic energy effect of the $n p$ mass difference) and concluded that about $46 \mathrm{keV}$ of the $116 \mathrm{keV}$ needed could be supplied by the sum of these small effects leaving about $70 \mathrm{keV}$ to be attributed to further CSB interactions. Friar, Gibson and Payne [85] found a very similar result.

They estimate that a potential which represents the shift of scattering lengths in the singlet S state of $-17.5 \mathrm{fm}$ to $-18.6 \mathrm{fm}$ (see section IIIC for a discussion of the source of these determinations) will also give the right value for the binding energy difference. The $\rho$ - $\omega$ CSB potential [80] provides a large fraction of what is needed in their calculation. For the breaking of charge independence they assumed a phenomenological Woods-Saxon potential with a radius of $0.5 \mathrm{fm}$, a diffuseness of $0.2 \mathrm{fm}$, and a $6 \mathrm{MeV}$ depth. The calculation is complicated by the fact that the absolute binding energy of ${ }^{3} \mathrm{H}$ is not given correctly so that an extrapolation must be made.

\section{NN Scattering states}

Scattering states are sensitive to different aspects of the breaking potentials than the bound states so, in principle at least, additional information can be obtained by studying scattering cross sections.

There is a wide range of values the ${ }^{1} \mathrm{~S}_{0} \mathrm{NN}$ scattering length that have been obtained experimentally (Table II), besides the $n p$ singlet scattering length first measured long ago in $n p$ scattering from ortho- and para-hydrogen [86 88]. It was found to be definitely negative leading to the conclusion that there was no lightly bound state of two neutrons. The study of the differences in these values has been the subject of many papers.

The $p p$ scattering length, $-17.3 \pm 0.4 \mathrm{fm},[74]$ is obtained from the removal of the Coulomb interaction in $p p$ scattering [89], a procedure which depends on the strong interaction used and the assumption of the form for the short range Coulomb potential. If this number is taken to represent the unbroken value then it is being assumed that all of the breaking in the proton-proton $(p p)$ scattering is due to the conventional Coulomb interaction.

Neutron-neutron $n n$ scattering lengths with values around $-16.1 \sim-16.3 \mathrm{fm}$ obtained from the final state interaction in $n d$ breakup were considered to be the recommended values at one time but now the value, $-18.5 \pm 0.3 \mathrm{fm}$, mostly from the $\pi^{-} d \rightarrow n n \gamma$ reaction is favored by the majority of physicists working in this field. This preference may be because the values from $n d$ breakup have a large dispersion and those from radiative pion absorption experiments are consistent [99] but also because the values around $-18 \mathrm{fm}$ 
are in the correct direction relative to $a_{p p}$ to explain the binding-energy difference in the three-nucleon system.

The value extracted from the $n p$ interaction is far from the group of the others. Since this value indicates a system closer to being bound it would seem to require an additional attractive potential (relative to the others) to move the value in that direction.

The breaking expressed by the difference of the value of the $n p$ scattering length compared with the average of the $n n$ and $p p$ values is usually considered as a separate effect (charge independence breaking, CIB) from the charge symmetry breaking (the difference between $a_{n n}$ and $a_{p p}$ ). Since only neutral pions can be exchanged in $n n$ and $p p$ scattering, the fact that the charged pion mass is different from that of the neutral pion does not enter into CSB. However, it does contribute to the CIB. The replacement form for one-pion exchange when the masses are different was worked out some time ago [103, 104] and has been claimed to provide much [105] or even all [106] of the breaking needed. Cheung and Machleidt [105] point out that Ericson and Miller [106] used an approximation which, when corrected, reduces their value to something comparable with other determinations. All of these corrections were calculated without form factors or with form factors with large regulating masses. We find that this correction is sensitive to the value of the cut-off parameter as will be discussed later in the text.

The role of two pion exchange in CIB has been the subject of several studies, resulting in a wide range of values for the contribution to the difference from the average $n n$ and $p p$ values of the singlet $n p$ value. Some values are: $0.65 \mathrm{fm}$ [107], 0 [104], $0.16 \mathrm{fm}$ [108], $0.18 \mathrm{fm}$ [105], and $0.88 \mathrm{fm}[106$.

The pattern of these scattering lengths, which should all be the same under $\mathrm{SU}(2)$ symmetry, would seem to require three isospin-breaking interactions: one to connect the unbroken value to the $p p$ scattering, one to connect it to the $n n$ scattering and a third to connect it to the singlet $n p$ scattering. One might suppose that the isospin-pure value would lie somewhere near the centroid of these values which would mean that the correction for the $p n$ case would be represented by a attractive potential while the $n n$ scattering would be produced by a repulsive potential. The $p p$ potential would have to be more repulsive than the $n n$ case to give the right sign for the mirror nuclei difference observed. The considerations in the following sections naturally lead to three potentials which fulfill these conditions.

\section{A challenge to the meson-exchange theory of CSB}

This picture seems to have changed when Goldman, Henderson and Thomas [109] calculated the effect of a form factor in the derivation of the CSB $\rho-\omega$ potential in a quark picture and found that the resulting potential is much smaller than previously believed. While one might question the quark model used, their paper was shortly followed by one by Piekarowicz and Williams [110] using hadronic $(N \bar{N})$ intermediate states which found qualitatively the same result and one by Hatsuda et al. [111] which found a similar result using QCD sum rules. Soon after followed several more papers which argued that these calculations were only examples of a general rule that the contribution to CSB from $\rho$ - $\omega$ mixing should be negligible [112 116] (but see [117, 118]). Thus, it appears that a replacement theory is needed.

One such alternative theory was the treatment of the effect of nucleon mass differences in two-pion exchange. The major contributor is the crossed diagram for the exchange of two charged pions with nucleon or $\Delta$ intermediate states. The difference of the internal 
nucleon masses, compared with the external masses, changes sign for $n n$ compared with $p p$ scattering.

Coon and Niskanen [119] found that the difference in scattering lengths could be understood with this effect in spite of an earlier calculation which had found the effect to be 3-5 times smaller. Li and Machleidt [108] treated the same diagrams and also found that they could understand the symmetry breaking. However, the two calculations are very different. Coon and Niskanen [119] find that the diagram with two nucleons in the intermediate state dominates while $\mathrm{Li}$ and Machleidt [108] find that it is the diagram with one $\Delta$ which is, by far, the largest. Machleidt and Mütter [120] investigated the possibility of choosing between the heavy meson exchange contribution and the two-pion exchange by studying the partial waves with $\ell>0$.

The paper by Coon and Niskanen [119] contains a summary of the calculations of the two-pion exchange (before the work of Li and Machleidt) and concludes that "This cannot be considered a satisfactory theoretical situation." These calculations were done with regulating masses of the pion form factor of the order of $1 \mathrm{GeV}$. Coon and Niskanen [119] show that the scattering length difference is sensitive to the value chosen. For the values of this mass that we use (see section IVE) the contribution of these diagrams would be very small.

\section{COULOMB EFFECTS DUE TO CLUSTERING}

\section{A. Intra-nucleon Coulomb}

First we consider the Coulomb energy which arises from the assembly of several charges. The total energy contained in such a charge cloud depends very much on the details of how the charges are clustered. An example is provided by the nucleons themselves.

The proton has two valence quarks $(u)$ with charge $+2 / 3$ and one $(d)$ with charge $-1 / 3$. If they are equidistant from each other with an average inverse distance of $\left\langle\frac{1}{d}\right\rangle$ then the total coulomb energy of the system is

$$
e^{2}\left[\frac{2}{3} \times \frac{2}{3}-\frac{1}{3} \times \frac{2}{3}-\frac{1}{3} \times \frac{2}{3}\right]<\frac{1}{d}>=0
$$

For the neutron we have

$$
e^{2}\left[\frac{1}{3} \times \frac{1}{3}-\frac{1}{3} \times \frac{2}{3}-\frac{1}{3} \times \frac{2}{3},\right]<\frac{1}{d}>=-\frac{1}{3} e^{2}<\frac{1}{d}>.
$$

This result is perhaps less surprising when one considers that an alternate decomposition, $n \rightarrow p+\pi^{-}$(the pion cloud), would give a negative Coulomb energy as well. There is no reason, however, to believe that all of the distances between quarks are the same, indeed one popular model has two of the quarks combined in a diquark which has a small size. Of course, this effect still has the wrong sign to explain the $n p$ mass difference; invoking fundamental quark mass differences appears to remain necessary.

This form of interior Coulomb effect has been known for a long time and forms the basis for predictions of the electromagnetic mass differences of Baryons and Mesons (see, for example, [140 142] and [72] and references therein. 


\section{B. Coulomb effects interior to the confinement range of two nucleons}

When two nucleons are at small values of the center-of-mass separation the six quarks intermixed give more possibilities for correlations among them leading to additional Coulomb-energy effects. We restrict this freedom by correlating two of the quarks in each nucleon to represent a diquark (D). The view that we have is that when the two confinement regions touch, and begin to overlap, the partons can move around in the combined volumes. The initially unpaired quarks will be able to pair up and form a diquark correlation. Of course this will happen only a fraction of the time so that some probability will be associated with the event. It is plausible that the motion of these components will be governed by the interaction with the gluons within the expanded confinement region so that the pairing will be largely independent of the initial state of the nucleons and the breaking will be state independent. Of course, within a nucleus, states which differ in having a different probability for the overlap of the nucleons would show a state dependence.

To obtain an estimate of the scale of Coulomb energies for a single diquark, consider using an exponential shape for the density of the relative motion of the two quarks forming the diquark. In this case we have

$$
E_{C}=\frac{1.44 \sqrt{3} n}{<r^{2}>^{\frac{1}{2}} 9} \approx \frac{0.277 n}{<r^{2}>^{\frac{1}{2}}} \mathrm{MeV},
$$

where $<r^{2}>^{\frac{1}{2}}$ is the r.m.s. radius of the correlation density in fm (from 0.25 to $1 \mathrm{fm}$ from different models) and $n=+4,+1$ and -2 for $u u, d d$, and $u d$ pairs.

Any model for Coulomb energy due to quark clustering in the two-nucleon system will have (at least) three physical parameters:

1) The overall size of the system, expressed here as the diameter of a six quark bag. This parameter will control the size of the Coulomb effect between the uncorrelated quarks and the range of the resulting interaction. Representing the combined system as a sphere is a crude approximation, since, as the interaction proceeds, one would expect a deformation of the individual confinement regions as well as a possible "necking" of the combined system.

2) The range of the correlations. This (along with the electric charges) controls the Coulomb energy contained in each correlated quark pair.

3) The probability of forming a quark-quark correlation. This can be expressed as the probability of the two-quark state being found in a given configuration, given that it is known to be found in a known volume.

The following model contains these three pieces of physics in a simplified form. While the illustrative model to be presented shortly does not assume point diquarks, it is useful in order to get a qualitative picture to consider this example. In this case one has four clusters of charge to deal with. The result depends on which two quarks are taken to form the diquark.

A common assumption (which we shall follow) is to take the unlike quarks in the nucleon to form the diquark (one $u$ and one $d$ giving a charge $+1 / 3$ object). In this case one has for the $\mathrm{CC}$ interaction between two neutrons

$$
C_{n n}(r)=e^{2}\left(\frac{1}{3} \times \frac{1}{3}<\frac{1}{d}>_{D D}+\frac{1}{3} \times \frac{1}{3}<\frac{1}{d}>_{q q}-\left(\frac{1}{3} \times \frac{1}{3}+\frac{1}{3} \times \frac{1}{3}\right)<\frac{1}{d}>_{D q}\right)
$$


$B$ Coulomb effects interior to the confinement range of two nucleons

$$
=\frac{e^{2}}{9}\left(<\frac{1}{d}>_{D D}+<\frac{1}{d}>_{q q}-2<\frac{1}{d}>_{D q}\right)
$$

where $\left\langle\frac{1}{d}>_{D D}\right.$ is the average inverse distance between the diquarks in the two nucleons, $\left.<\frac{1}{d}\right\rangle_{q q}$ is the average inverse distance between the non-associated quarks in each nucleon (we will assume shortly that this pair of quarks also forms a diquark but for the moment it is general) and $\left\langle\frac{1}{d}\right\rangle_{D q}$ is the average inverse distance between the odd quark in one nucleon and the diquark in the other. There are of course other Coulomb energies from quarks in the interior of each nucleon but these do not depend on the distance between the centers of mass of the two nucleons (or so we will assume) and does not contribute to the crypto-Coulomb potential.

For a neutron and a proton we have

$$
\begin{gathered}
C_{n p}(r)=e^{2}\left(\frac{1}{3} \times \frac{1}{3}<\frac{1}{d}>_{D D}-\frac{2}{3} \times \frac{1}{3}<\frac{1}{d}>_{q q}+\left(\frac{1}{3} \times \frac{2}{3}-\frac{1}{3} \times \frac{1}{3}\right)<\frac{1}{d}>_{D q}\right) . \\
=\frac{e^{2}}{9}\left(<\frac{1}{d}>_{D D}-2<\frac{1}{d}>_{q q}+<\frac{1}{d}>_{D q}\right)
\end{gathered}
$$

and

$$
\begin{gathered}
C_{p p}(r)=e^{2}\left(\frac{1}{3} \times \frac{1}{3}<\frac{1}{d}>_{D D}+\frac{2}{3} \times \frac{2}{3}<\frac{1}{d}>_{q q}+\left(\frac{1}{3} \times \frac{2}{3}+\frac{2}{3} \times \frac{1}{3}\right)<\frac{1}{d}>_{D q}\right) \\
=\frac{e^{2}}{9}\left(<\frac{1}{d}>_{D D}+4<\frac{1}{d}>_{q q}+4<\frac{1}{d}>_{D q}\right)
\end{gathered}
$$

for the two proton case. Clearly the "ordinary Coulomb potential between two protons is included in this last expression as well.

The dependence on $r$, the center-of-mass separation of the nucleons, comes from the dependence of the average inverse of the distance between quarks on it.

If one assumes that the odd quarks (in different nucleons) become correlated so as to have a large inverse distance, one can obtain the negative potential needed for the $n p$ scattering length. A moderately strong effect is also due to interaction in the $D D$ system. However, it is the same for all nucleon pairs so does not make a contribution to the breaking. Neglecting $<\frac{1}{d}>_{D q}$ we see that $C_{n p}(r)$ is attractive (making the scattering length more negative than the unbroken case) while both $C_{n n}(r)$ and $C_{p p}(r)$ are repulsive (making their scattering lengths less negative). Of course it is well known that the difference in the neutral and charged pion masses makes a significant contribution to the CIB. This effect is discussed in section IVE.

\section{Schematic model}

For our purposes we do not need to know the wave function of the quarks since the density is enough to calculate the Coulomb energies. We consider a (modest) range of diquark sizes and vary the strength of the correlation to fit the $n p$ singlet scattering length. We make use of Ockham's razor in the calculation by not discussing any feature that is not directly relevant.

We neglect any isospin breaking in the strong interaction; all violations come from these crypto-Coulombian potential energies. Thus, there is a single density which is the same for 


\begin{tabular}{|cccc|}
\hline quark pair & $p n$ & $n n$ & $p p$ \\
\hline $1-4$ & +4 & +4 & +4 \\
$1-5$ & -2 & -2 & -2 \\
$2-4$ & -2 & -2 & -2 \\
$2-5$ & +1 & +1 & +1 \\
\hline $1-6$ & +4 & -2 & +4 \\
$2-6$ & -2 & +1 & -2 \\
$3-4$ & -2 & -2 & +4 \\
$3-5$ & +1 & +1 & -2 \\
\hline $3-6$ & -2 & +1 & +4 \\
\hline
\end{tabular}

TABLE II: Multiple of the factor 1/9 for the charges on individual quarks. Quarks 1 and 2 are members of a diquark in one nucleon and 4 and 5 form a diquark in the other while 3 and 6 are unpaired in the separated nucleons but are correlated when the nucleons overlap. The model expectation value for the inverse distances is the same within each group (between the lines) allowing the addition of the coefficients, resulting in the same values as in Eqs. 4, 5, and 6 .

any singlet NN pair, only the charges of the quarks are varied according to the particular nucleon pair.

One can look ahead to anticipate the results of the model qualitatively before making the calculation. Since the interaction of the members of the intranucleon diquarks are all the same and the interactions of the odd quark in one nucleon with the members of the diquark in the other nucleon are also all the same, the interactions can be grouped as shown in Table II. This grouping leads us back to Eqs. 4, 5] and 6 with the interpretation of the average inverse distances corresponding to the groups in Table II. Thus, we need only calculate the three inverse distances, which are the same for all nucleon pairs, as a function of $r$ and use the combinations given in the table to get an estimate of the $\mathrm{CC}$ contribution for each case. From this the relative contributions can be calculated immediately. We first note that the $D D$ contribution (first term) is the same for all nucleon pairs so leads to a shift away from the isospin conserved value which is the same for all nucleon pairs and hence is not visible in the differences among nucleon pairs that are observed experimentally.

The Coulomb interaction between the odd quark in one nucleon with the diquark in the other nucleon (third term in the equations, group two in the Table) is relatively small since no correlation between them is assumed. Thus the interaction between the two odd quarks will dominate. We can then get an estimate of the relative size of the various breaking potentials from the coefficients. The $n p$ potential will have an attractive potential with weight two and the $n n$ potential will have a repulsive potential with weight one giving the breaking between $n n$ and $n p$ a weight of three. The $p p$ potential will have a weight of four so that the breaking between the $p p$ and $n p$ systems will have a weight of six or twice the difference between the $p p$ and $n n$ potentials. Of course the pion mass difference also contributes to the CIB. The factor of four in the $p p$ case is less clean than assumed above because the comparison is usually made with the "Coulomb corrected" value of the $p p$ scattering length and hence depends on the form of the Coulomb potential used at short range.

Since both scalar and axial vector versions of diquarks have been proposed, the nucleon wave function might consist of a linear combination of the two. We don't need to know that, however. We only need to know that there is a quark-quark correlation between the odd quarks in the two-nucleon system and it needs to be the same for all nucleon 
pairs to have the amplitude due to the strong interaction be invariant under $\mathrm{SU}(2)$. The correlation which is assumed to form between the two odd quarks need not have the same properties as the correlation already present in the individual nucleons, although we assume here that it does to reduce the number of parameters. The correlation between the odd quarks is assumed to always be the same whether they are like or unlike quarks (which might seem to indicate that the correlation is spin-one). Without that assumption a large breaking of isospin symmetry would be present in the strong interaction.

We now present a representative model calculation based on the naïve quark model considering only the valence quarks. While this model is too crude to believe in any detail, one may hope that the basic physics is contained in this simple form. There are no dynamics in the model; it is represented by a static quark density. The diquark in this case is embodied by a correlation between two of the quarks in each nucleon and a correlation representing the formation of a diquark consisting of the combination of the remaining unpaired quarks in the initial nucleons. We are considering the scattering of very low energy nucleons so the time available for this formation is very long and the combined two-nucleon system is considered as a static object.

The density is expressed as a product of correlation functions. The quarks are considered as distinguishable and are numbered 1 through 6. Quarks 1, 2, and 3 are in one nucleon and 4,5 and 6 are in the other. Confinement is represented by a limit of the distance between pairs of quarks. More complicated (and more realistic?) conditions could easily be included. The confinement and correlation functions are the same for all NN pairs and are as follows:

pairs 1-2 and 4-5 are confined and correlated,

pairs 1-3, 2-3, 4-6, 5-6 are confined but not correlated,

pair 3-6 (the odd quarks) are correlated but not confined,

all other quark pairs have no constraints.

The correlation is represented by a Jastrow style factor

$$
F_{c}(d)=1+\alpha e^{-\beta d}
$$

where $d$ is the distance between two quarks and $\alpha$ is defined in terms of $\beta$ by

$$
\alpha=\frac{1}{2} p \beta^{3}
$$

thus adjusting the normalization so as to compensate for the range of the correlation. It is the parameter $p$ which is varied. With this choice of normalization, $p$ remains of the order of $1-5 \mathrm{fm}^{3}$ as we will see later.

As discussed above we assume that the correlation between "odd" (unpaired) quarks in different nucleons is the same although they form a $u d$ pair in the $n p$ case and a $u u$ or $d d$ pair in the cases of like nucleons.

The confinement factor is

$$
G(d)=\frac{1}{1+e^{(d-c) / a}}
$$

where $a$ is chosen to be very small $\left(10^{-4} \mathrm{fm}\right)$. The length $c$ represents the diameter of a spherical bag containing the 6 quarks.

Now that the model nucleon density has been defined, the breaking potential can be calculated by taking the expectation value of the Coulomb energy over the density for fixed chosen values of the center-of-mass distance (the usual adiabatic approximation) 
which we have already remarked can be reduced to the calculation of the average of three inverse distances. The calculation needed is an 18 dimensional ( 6 particles in 3 dimensions) integral over a highly correlated integrand with the normalization to be determined. It is likely that several judicial changes in integration variables would permit a reduction in the number of dimensions (perhaps even considerably) but we treat the integral as it stands with the Metropolis algorithm, which could still be applied in a more general case (say, with a truly 3 -body confinement formula). This method also readily permits specifying the distance between the centers of mass.

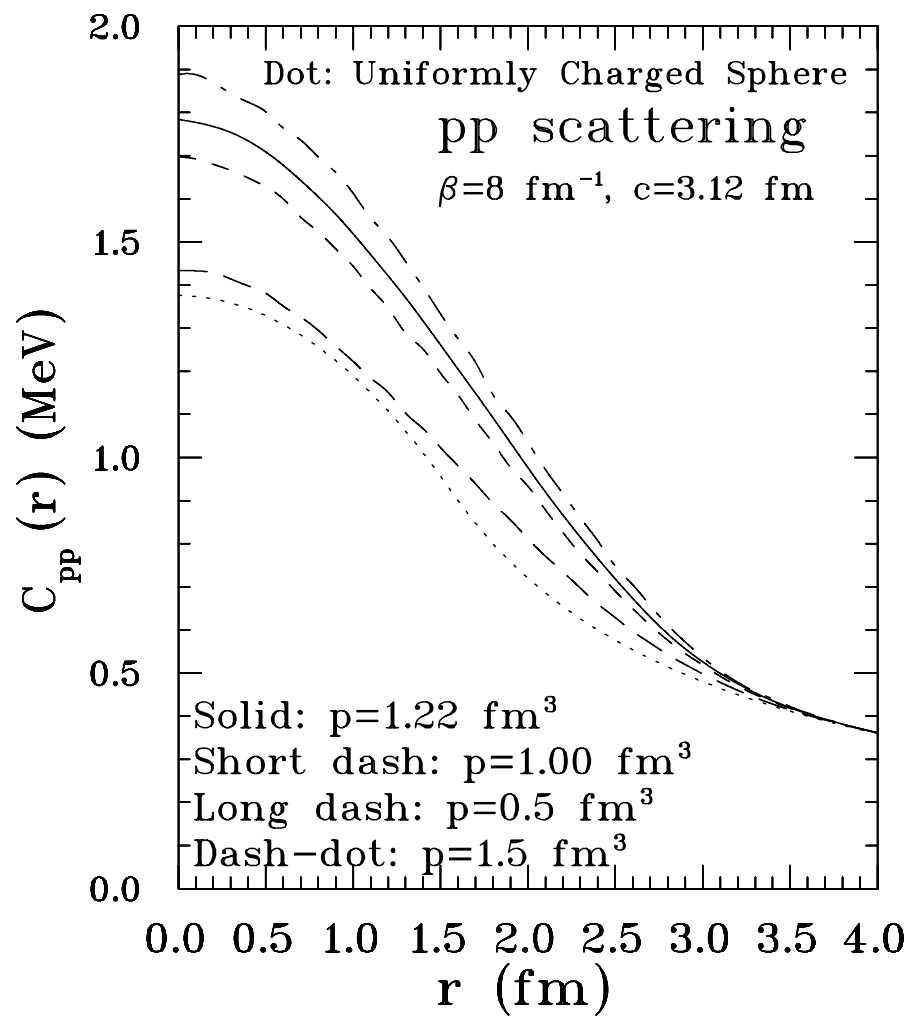

FIG. 2: Variation with parameters of the model for the full $p p$ Coulomb potential.

The Metropolis algorithm creates a list of configurations (walkers) which consists of sets of the 18 coordinates giving the possible positions of all of the particles. The frequency of the appearance of a given configuration (or similar configurations) gives the probability for that configuration. In order to fix the distance between the centers of mass it is sufficient to restrict the possible walkers to those with the desired separation. To do this, the two nucleons are created with their centers of mass located at the origin at each Metropolis step. Then, formally, one nucleon is translated from the origin by the center-ofmass separation specified for any given calculation. In practice this translation can be combined with the evaluation of the distances needed for the calculation of the walker coordinates. The distances between quarks in the same nucleon are given by $\mathbf{r}_{i}-\mathbf{r}_{j}(i, j$ in the same nucleon) while the distances between quarks in different nucleons are given by $\mathbf{r}-\mathbf{r}_{i}+\mathbf{r}_{j}$ where $\mathbf{r}$ is the distance between the centers of mass of the two nucleons, $i$ is 1,2 , or $3: j$ is 4,5 , or 6 and the conditions $\mathbf{r}_{1}+\mathbf{r}_{2}+\mathbf{r}_{3}=0$ and $\mathbf{r}_{4}+\mathbf{r}_{5}+\mathbf{r}_{6}=0$ are maintained throughout the calculation. The quark mass differences that would be needed to explain the $n p$ mass difference are not included in the present calculation. Thus there is no $\mathrm{SU}(2)$ breaking in the strong interaction in the model; all of the breaking comes from the Coulomb interaction.

The calculation was carried out with a Metropolis step size of $0.03 \mathrm{fm}$ with 4000 walkers and 200,000 steps. The calculation for one value of the relative distance between centers of mass requires 25 minutes on a $2.5 \mathrm{GH}$ CPU. With a 4 core processor the calculation of the CC potential to $4.0 \mathrm{fm}$ in steps of 0.1 fm takes less than 5 hours

Figures 2, 3] and 4 show some examples of the potentials obtained with the model which result from the Coulomb interaction between the correlated quark pairs. The $p p$ potential in Fig. 2 includes the full Coulomb potential (CC+ "ordinary"). 
E Contribution of the difference of pion masses to the breaking of charge independence

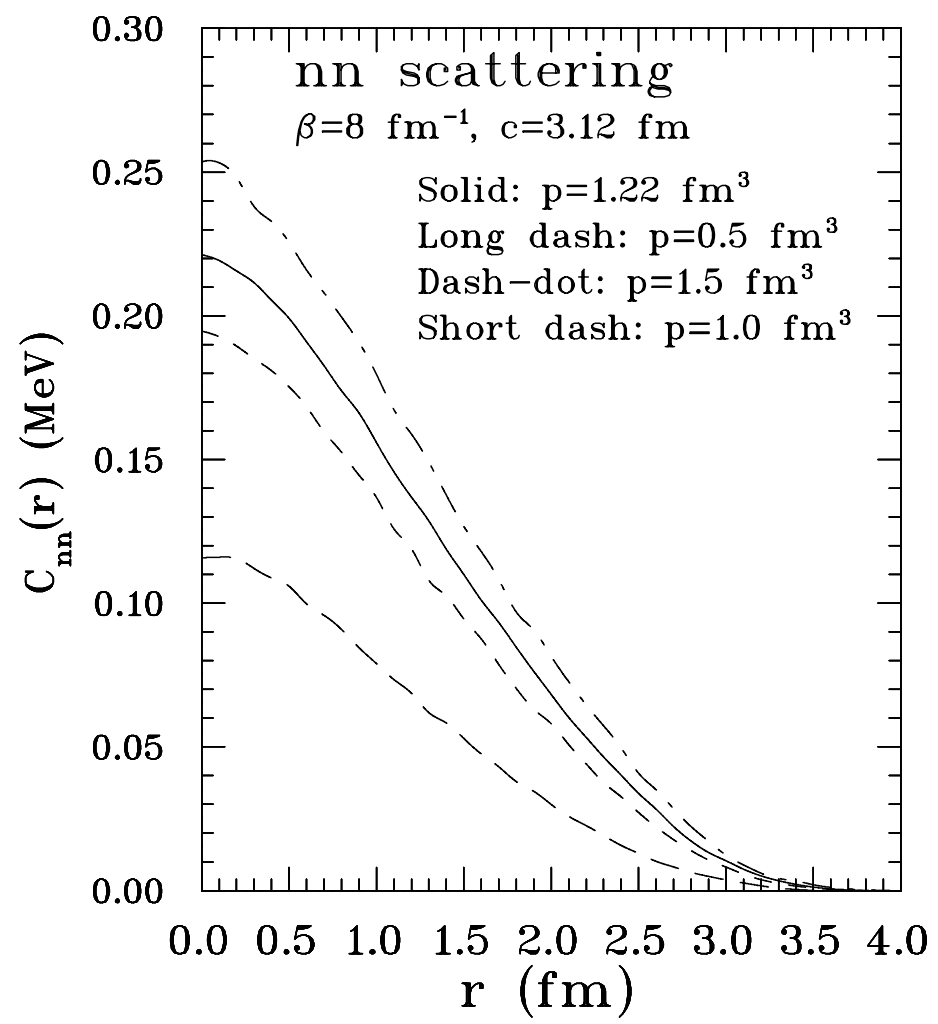

FIG. 3: Variation with parameters of the model for the $n n$ crypto-Coulomb potential.

\section{Completing the potential}

In order to actually compare with the data, full potentials must be available. It is assumed that there must be an isospin pure strong-interaction potential which is the same for all three singlet-nucleon pairs. The parameters of this potential are determined by fitting the accurate $p p$ data including the full Coulomb (both ordinary and $\mathrm{CC}$ ) potential for the $p p$ case.

An isospin invariant potential given (in MeV) by a form similar to the one assumed by Reid [10],

$$
V_{0}(r)=\frac{-C_{1} e^{-\mu_{1} x}+C_{2} e^{-\mu_{2} x}}{\mu_{0} r}-10.47 \frac{\left(e^{-\mu_{0} r}-e^{-M r}\right)}{\mu_{0} r}
$$

is employed for the $n n$ and $p p$ cases. The values $\mu_{1}=4 \mathrm{fm}^{-1}$ and $\mu_{2}=7 \mathrm{fm}^{-1}$ were used by Reid [10] and we have kept the same ranges except where specified otherwise. Here $x=0.6945 r, \mu_{0}=0.6840$ is the neutral pion mass in $\mathrm{fm}^{-1}$, while $M$ is the mass governing the square of the pion-nucleon off-shell form factor (see discussion below). For the $n p$ interaction where charged pion exchange contributes, the third term is replaced by [103, 104]

$$
10.47\left[\frac{\left(e^{-\mu_{0} r}-e^{-M r}\right)}{\mu_{0} r}-2\left(\frac{\mu_{+}}{\mu_{0}}\right)^{2} \frac{\left(e^{-\mu_{+} r}-e^{-M r}\right)}{\mu_{0} r}\right]
$$

where $\mu_{+}$is the mass of the charged pion.

\section{E. Contribution of the difference of pion masses to the breaking of charge independence}

The difference in mass between the charged and neutral pions has long been recognized 103, 104 as an important contributor to the breaking of charge independence. Coon and Scadron [104] found that mass differences in the exchange of heavy mesons and two-pion exchange generated only small breaking effects. We find that the degree of breaking from the pion mass difference depends rather sensitively on 


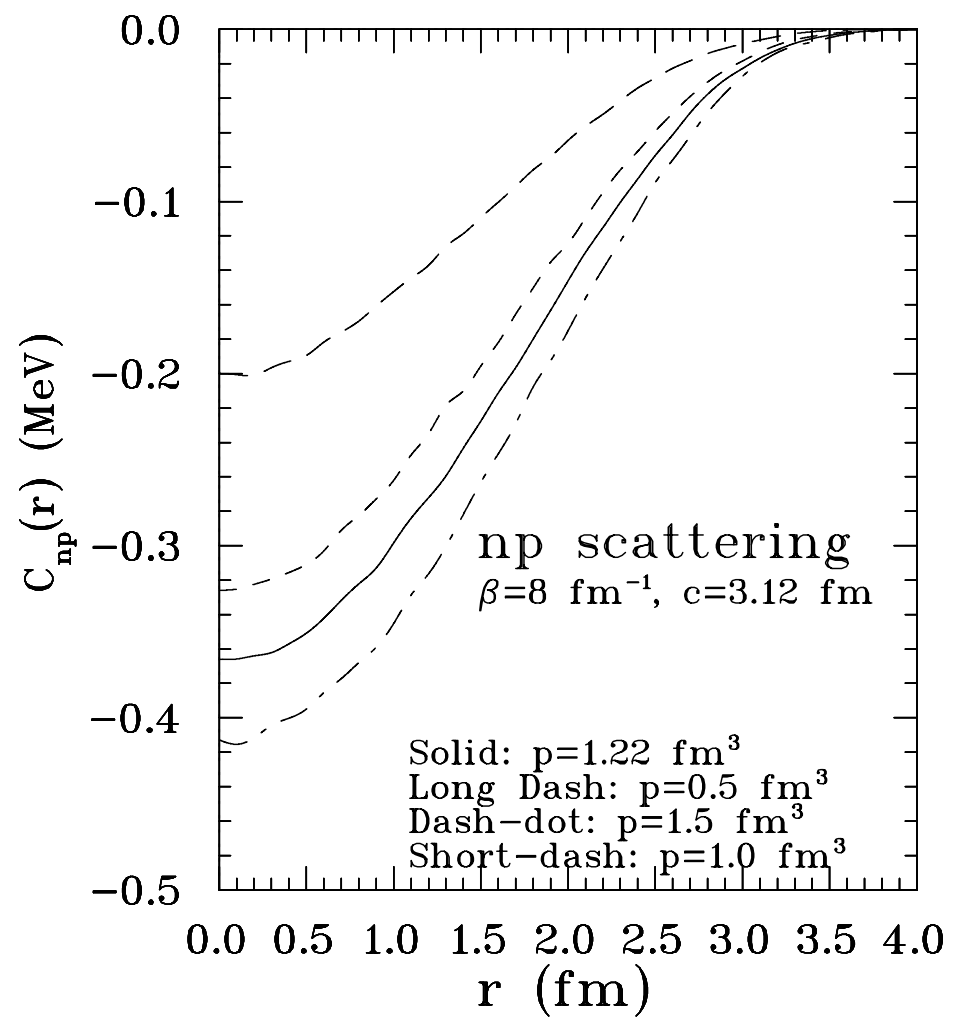

FIG. 4: Variation with parameters of the model for the $n p$ crypto-Coulomb potential.

the off-shell form factor. The form factor lowers the strength of the one-pion-exchange (OPE) potential at short distances and since we require that the total potential must be such that the $p p$ scattering data are correctly fit, the fraction of OPE potential becomes less and the breaking due to the pion mass difference along with it.

The correction for the finite size of the pion-nucleon system is often expressed as a vertex function. If this vertex function is denoted by $v(q)$ then the modified one-pion-exchange potential will be given by

$$
V_{\pi}(r)=\frac{f^{2}}{4 \pi} \int_{0}^{\infty} q^{2} d q \frac{j_{0}(q r)}{q^{2}+\mu^{2}} v^{2}(q) .
$$

The expressions Eqs. (10/11) above result from taking

$$
v^{2}(q)=\frac{M^{2}-\mu^{2}}{q^{2}+M^{2}}
$$

To compare with values of $M$ taken from the literature some correspondence must be made. In no case is an exact comparison possible. Often one compares form factors by making an expansion for small $q$ and matching the coefficients of $q^{2}$. While it is not clear that this is the proper procedure, since it corresponds to comparing at large $r$ and we are interested in the behavior at small $r$, we will often follow that method as well.

The pion form factor reduces the strength of the potential at small values of $r$. For the form mentioned above

$$
\frac{1}{\mu^{2}+q^{2}} \frac{M^{2}-\mu^{2}}{M^{2}+q^{2}} \rightarrow \frac{\pi}{2} \frac{\left(e^{-\mu r}-e^{-M r}\right)}{r} .
$$

The proper value of the pion-nucleon form factor has been much debated over the years $([121-127]$. A survey of this early literature leads us to values of $M$ is the range of 1.4 to $2.8 \mathrm{fm}^{-1}$.

Recently there has been a great deal of interest in the development of an effective field theory in which the dependence on the form factor range would disappear at the cost of a renormalization order by order. While this program has met with partial success [128], an observable dependence on the range remains. 
In a recent high-quality fit to the NN phase shifts, Entem and Machleidt [129] used a Gaussian form factor with $\Lambda_{g}=0.5 \mathrm{GeV}$. When using potentials of this type to construct the Equation of State of neutron matter [130, 131], Gaussian form factors with a range for $\Lambda_{g}$ of $450 \mathrm{MeV} / \mathrm{c} \leq \Lambda_{g} \leq 650 \mathrm{MeV} / \mathrm{c}$ were used. Epelbaum, Glockle and Meissner used $\Lambda_{g}$ around $600 \mathrm{Mev} / \mathrm{c}$ in N $\mathrm{N}^{3} \mathrm{LO}$ calculations [132]. Epelbaum and Meissner suggest [133] that $\Lambda_{g} \approx 3 \mathrm{fm}^{-1}$ is the value that should be used. Values of $\Lambda_{g}$ in the same range appear optimal in the strange sector [134 136] as well.

The form factor is often regarded as the Fourier transform of the density of elementary scattering centers in a composite target. With the assumption of a Gaussian density the form factor becomes

$$
S(q)=e^{-<r^{2}>q^{2} / 6}=e^{-q^{2} / \Lambda_{g}^{2}}
$$

Using $<r^{2}>^{\frac{1}{2}}=0.86 \mathrm{fm}$ (a common value for the charge radius of the proton) leads to $\Lambda_{g}=562$ $\mathrm{MeV} / \mathrm{c}$.

To attempt to match these Gaussian form factors to the one we have used, one can consider several possibilities. From the expansions for small $q$ one gets $M=\Lambda_{g} / \sqrt{2}$. One might also choose to make the two form factors (or their squares) equal at some momentum scale $q_{0}$, i.e.,

$$
\frac{1}{1+q_{0}^{2} / M^{2}}=e^{-2 q_{0}^{2} / \Lambda_{g}^{2}}
$$

Choosing $q_{0}=M$ we find $M / \Lambda_{g}=\sqrt{\ln 2 / 2} \approx 0.588$ leading to $M=1.79 \mathrm{fm}^{-1}$ for $\Lambda_{g}=600 \mathrm{MeV} / \mathrm{c}$.

We remind the reader that there exists in the literature a least one example [137] where it was pointed out [138] that leaving out the pion-nucleon form factor in the calculation of the meson-exchange-current contribution to elastic electron scattering led to a wrong prediction (by a factor of 30) as verified by experiment.

Thomas and Holinde [139] pointed out that the apparent discrepancy between the charged and neutral $\pi N N$ coupling constants is resolved if the mass in a monopole for each vertex is in the range 500-800 $\mathrm{MeV}$ (corresponding to $M \approx 350-560 \mathrm{MeV}$ or $\approx 1.75-2.80 \mathrm{fm}^{-1}$.

In summary, the values quoted here from the literature lie mainly in the range $M=260-500 \mathrm{MeV} / \mathrm{c}$ (1.4 to $2.8 \mathrm{fm}^{-1}$ ). We adopt a value of $M=2 \mathrm{fm}^{-1}$ as representative of these limits.

\section{EXTRACTING THE “COULOMB CORRECTED” $p p$ PHASE SHIFTS}

We now give the method used to extract the pure $p p$ singlet $S$-wave phase shifts from the data. By adding the closed form for the Rutherford amplitude

$$
f_{R}(\theta)=-\frac{\eta}{2 k \sin ^{2} \frac{\theta}{2}} e^{i\left(2 \sigma_{0}-\eta \ln \sin ^{2} \frac{\theta}{2}\right)},
$$

and subtracting the corresponding partial-wave expansion, we can write the full amplitude as

$$
\begin{aligned}
f(\theta) & =f_{R}(\theta)+\frac{1}{2 i k} \sum_{\ell}(2 \ell+1) P_{\ell}(\cos \theta)\left(e^{2 i \delta_{\ell}}-e^{2 i \sigma_{\ell}}\right) \\
& =f_{R}(\theta)+\frac{1}{2 i k} \sum_{\ell}(2 \ell+1) P_{\ell}(\cos \theta) e^{2 i \sigma_{\ell}}\left(e^{2 i \delta_{\ell}^{e}}-1\right),
\end{aligned}
$$

where $\delta_{\ell}$ in Eq. (18) is the full phase shift obtained by matching the solution of the Schrödinger equation with the strong plus CC and standard Coulomb potential included. The $\sigma_{\ell}$ are the "Coulomb phase shifts" which result from the solution of the Schrödinger equation with a point Coulomb potential. Since for large $\ell, \delta_{\ell} \rightarrow \sigma_{\ell}$, this series converges.

In equation (19) the modified phase shifts $\delta_{\ell}^{e}$ (called the "electric phase shifts" by Heller [143])

$$
\delta_{\ell}^{e} \equiv \delta_{\ell}-\sigma_{\ell}
$$

go to zero for large $\ell$ and at zero energy where both $\delta_{\ell}$ and $\sigma_{\ell}$ diverge.

We are interested in the phase shifts, $\delta_{\ell}^{0}$, given by the strong interaction alone and $\delta_{\ell}^{c c}$, which results from the strong plus CC potential. It is the latter which should be used in the calculation of nuclear 


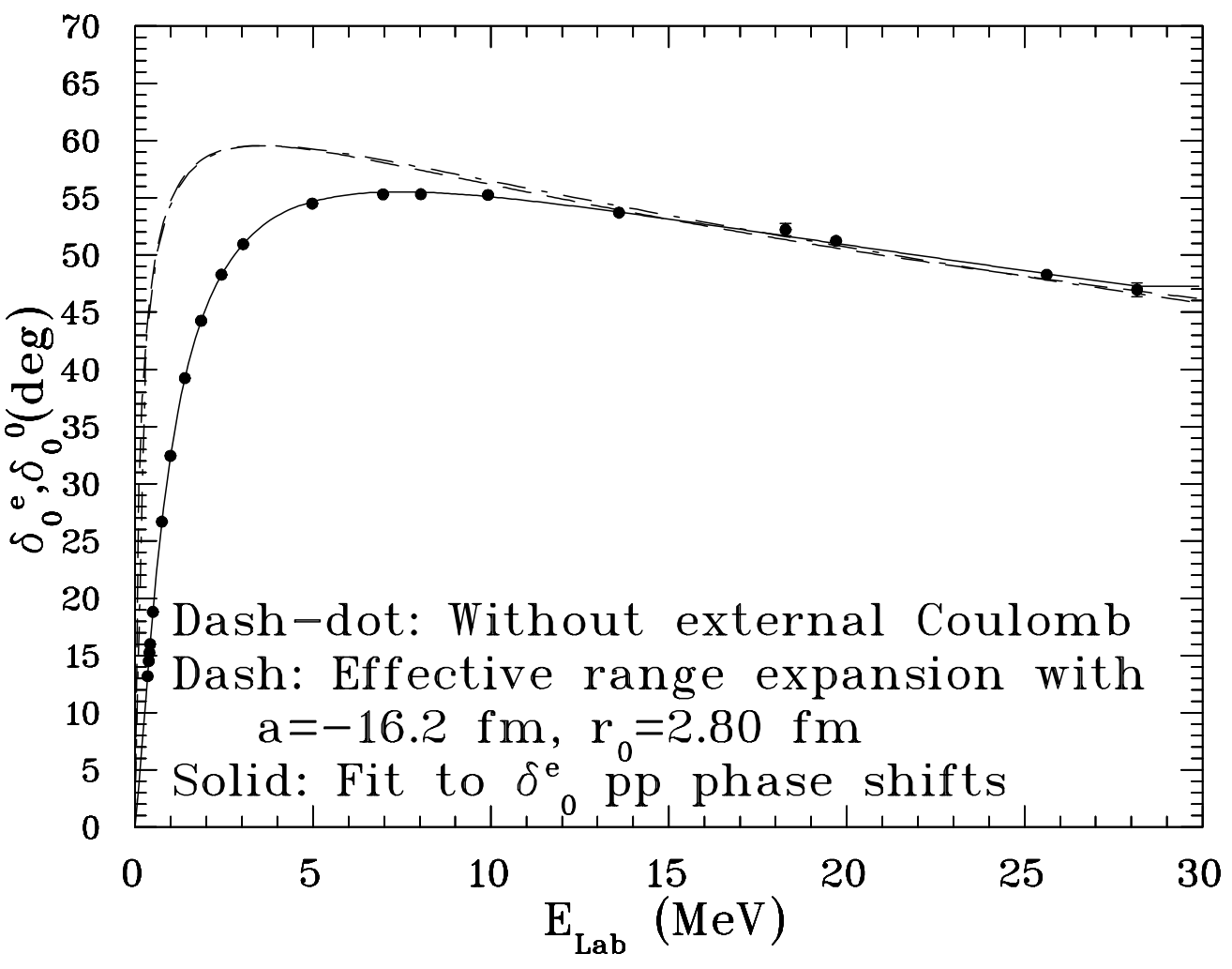

FIG. 5: Typical phase shift fit with the Reid-like potential (curve), [including the CC potential] to the values from Ref [89] (solid dots). This fit corresponds to the solid line in Fig. 2 The difference between the dash-dot and solid curves reflects the importance of the electric correction. The fitted model has $p=3 \mathrm{fm}^{3}$ and $\beta=4 \mathrm{fm}^{-1}$.

properties and the former which are to be considered as "isospin symmetric" results. In the limit that the standard Coulomb interaction goes to zero (so that $\delta_{\ell}^{e} \rightarrow \delta_{\ell}^{c c}$ ), this simple addition of phase shifts might be considered as the first order correction to the phase shift obtained with only the strong $+\mathrm{CC}$ interaction for the standard Coulomb effect, although $\delta_{\ell}^{e}$ clearly contains a further dependence on the standard Coulomb interactions. In order to calculate this additional dependence, models for the strong interaction are used. This correction is sometimes called the "inner Coulomb correction" in pion-nucleon scattering but we will call it the "electric correction" to avoid possible confusion with the CC effect. It only takes into account the Coulomb explicitly included in the Schrödinger equation, of course. Any hidden Coulomb effect (such as the crypto-Coulomb that we consider here) would not figure in this correction; it would appear as being part of the strong interaction.

The quantity $\delta_{\ell}^{e}$ can be obtained directly from the $p p$ data without recourse to any model [89]. For this reason it makes a good contact point for fitting models to the data. The precision of the data is among the best in nuclear physics: The low-energy points have uncertainties below $1 \%$, most less that $0.1 \%$ while those above $5 \mathrm{MeV}$ have uncertainties of the order of 0.2 to $1 \%$. We have added (in quadrature) uncertainties of $0.1 \%$ to the quoted values to take into consideration the crudeness of our model. We find that the data points are fit to within the order of $0.1 \%$ without difficulty (see Fig 5 ).

Turning off the entire Coulomb potential, including the CC part, we obtain $\delta_{0}^{0}$ and from it, the $a_{0}$ (isospin unbroken) scattering length. Keeping only the strong plus $p p$ CC potential we obtain the $p p$ version of $\delta_{0}$ and from it the value of $a_{p p}$ is obtained. By adding the attractive CC $n p$ potential (see Fig. (4) to $V_{0}, a_{n p}$ is obtained and with the repulsive $n n$ potential (Fig 3) $a_{n n}$ is found. This procedure was followed for the case of no pion mass difference and the physical difference between charged and neutral pion masses. The entire process was carried out for 3 different sizes of the diquark correlation range as expressed by $\beta$ and a sampling of values of the parameter $p$.

Figure 5 shows a fit (solid line) to the data. The dash-dot line shows the results of a calculation using the strong $+\mathrm{CC}$ potential only. One sees that above $15 \mathrm{MeV}$ the electric correction is very small, one would have obtained nearly the same values of $\delta_{0}^{c c}$ from a direct comparison with the data for $\delta_{0}^{e}$. The 
result of the effective range formula

$$
k \cot \delta_{0}=-\frac{1}{a}+\frac{1}{2} r_{0} k^{2}
$$

is also given (dashed line) and it is seen that the representation is very good. For $a=-16.2 \mathrm{fm}$ and $r_{0}=2.80 \mathrm{fm}$, the first term contributes $0.062 \mathrm{fm}^{-1}$ and (at $15 \mathrm{MeV}$ ) the second gives $0.25 \mathrm{fm}^{-1} \mathrm{so} \mathrm{that}^{-1}$ the effective range term dominates $(\geq 80 \%)$ at these energies and the fit is much more sensitive to $r_{0}$ than to $a$. Of course, at the very low energies the reverse is true.

\section{RESULTS}

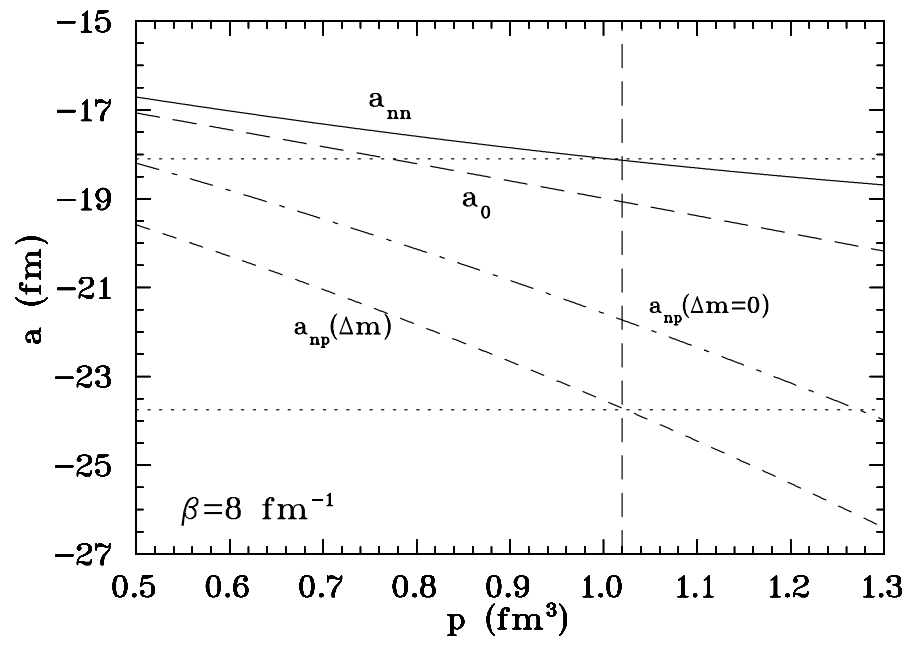

FIG. 6: Scattering length vs. the strength parameter $p$ for $\beta=8 \mathrm{fm}^{-1}$. The notation $\Delta m=0$ means that the difference in pion masses is not taken into account, the neutral pion mass is used in all one-pion exchange calculations. The notation $\Delta m$ means that the charged and neutral pion masses are used as in Eq. 11.

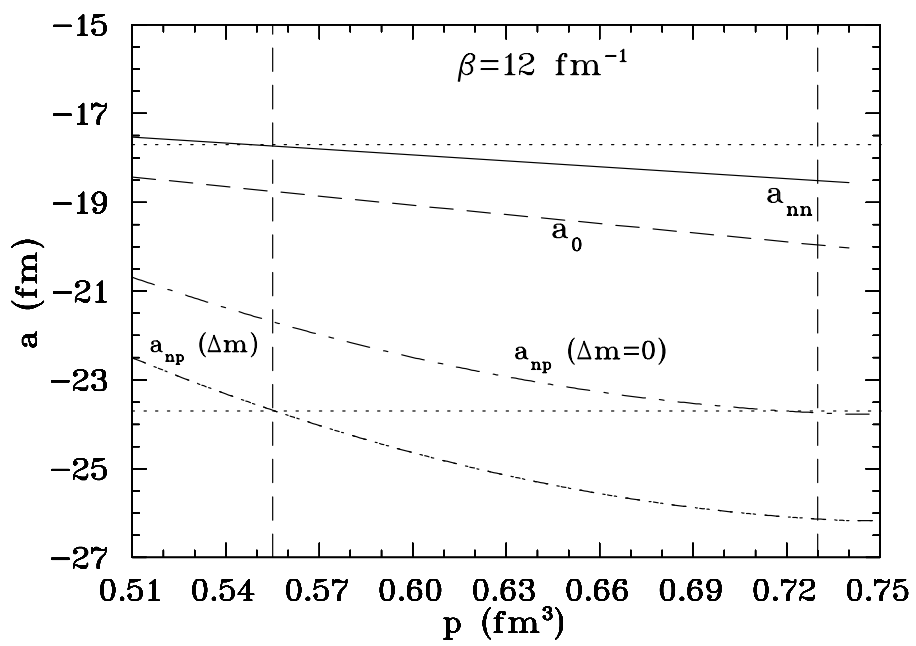

FIG. 7: Scattering length vs. the strength parameter $p$ for $\beta=12 \mathrm{fm}^{-1}$. See Fig 6 for notation. 


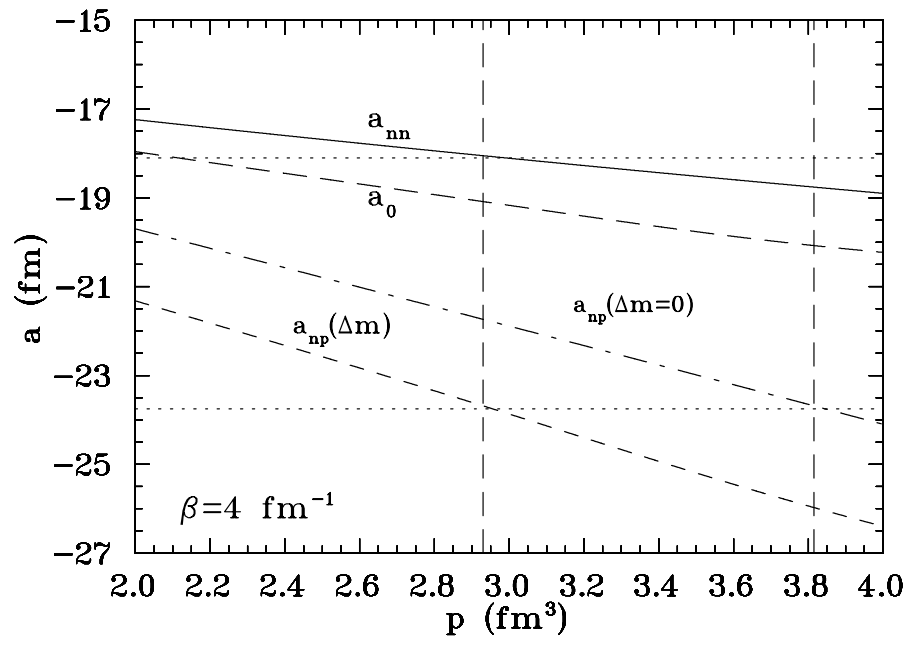

FIG. 8: Scattering length vs. the strength parameter $p$ for $\beta=4 \mathrm{fm}^{-1}$. See Fig 6 for notation.

\section{A. Relationship among the scattering amplitudes}

The curves in Figs. 6, 7 and 8 show how the amplitudes are related to each other and were obtained by the following procedure: The experimental $p p$ phase shifts, $\delta_{\ell}^{e}$ taken from reference [89] were fit using an isospin invariant strong potential (including one-pion exchange, $V_{0}(r)$ of Eq. (10) plus the full Coulomb potential (some examples are shown in Fig. 2) generated by the model for a set of values of the parameter $p$. As $p$ is varied the coefficients $C_{1}$ and $C_{2}$ must also change in such a way as to keep $V_{0}(r)$ plus the CC potential the same so as to fit the data. When the ordinary Coulomb potential, $V_{C}(r)$, is removed, as in the standard procedure, the resulting $a_{p p}$ calculated from this combined potential will be the same, or very nearly so. This potential would be considered as the "strong potential" if one were not aware of the presence of the CC potential. This compensation of variation of $V_{0}(r)$ and the CC potential it seen to be very good since the values of $a_{p p}$ are found to be (very nearly) independent of $p$ at $-16.2 \mathrm{fm}$.

As $p$ varies, however, $C_{1}$ and $C_{2}$ do change so that when $V_{0}(r)$ alone is used to calculate the pure isospin conserving scattering length, $a_{0}$, does change, as do $a_{n n}$ and $a_{n p}$ when calculated with their respective CC potentials.

To find the $n n$ scattering length $a_{n n}$ in these figures follow the short-dash curve until it crosses the experimental value of $a_{n p}$ (lower horizontal dotted line) to obtain the value of $p$ appropriate. Then the value of $a_{n n}$ can be read off at that value of $p$, for example in figure $6 . p=1.02 \mathrm{fm}^{3}, a_{n n}=-18.1 \mathrm{fm}$.

\section{B. Charge-symmetry-breaking Potentials}

The range of the correlation $r_{\text {corr }}=\sqrt{12} / \beta$ is $0.867 \mathrm{fm}$ for $\beta=4 \mathrm{fm}^{-1}, 0.433 \mathrm{fm}$ for $\beta=8 \mathrm{fm}^{-1}$ and $0.289 \mathrm{fm}$ for $\beta=12 \mathrm{fm}^{-1}$ compared with $0.27 \mathrm{fm}$ from Bloch et al. 7], $0.32 \mathrm{fm}$ from Carlson, Kogut and Pandharipande 9], $0.21 \mathrm{fm}$ from Sisodiya et al. [144], and $0.24 \mathrm{fm}$ from Keiner [28].

While it may at first appear that the order of the scattering lengths has changed since the $n n$ scattering length is now less negative than the unbroken one, the effect is the same as before since the CC potential for the $p p$ system is more repulsive than that of the $n n$ system so that there is an apparent stronger Coulomb effect in (say) the three-body system. One can take directly the difference of these potentials to find a charge symmetry breaking potential which could be used in the same way as the meson mixing potentials [80].

In general these potentials could be used directly in any calculation of nuclear properties. However, a charge symmetry breaking potential to be used in the same spirit as the $\rho-\omega$ potential discussed earlier in a perturbation calculation can also be obtained by removing the "ordinary" Coulomb potential (since the desired potential is to be beyond the standard Coulomb calculation) and then removing the interior repulsive potential of the $n n$ case. Figures 9, 10 and 11, show this progression and selected analytical fits to the breaking potentials are given in the appendix. 


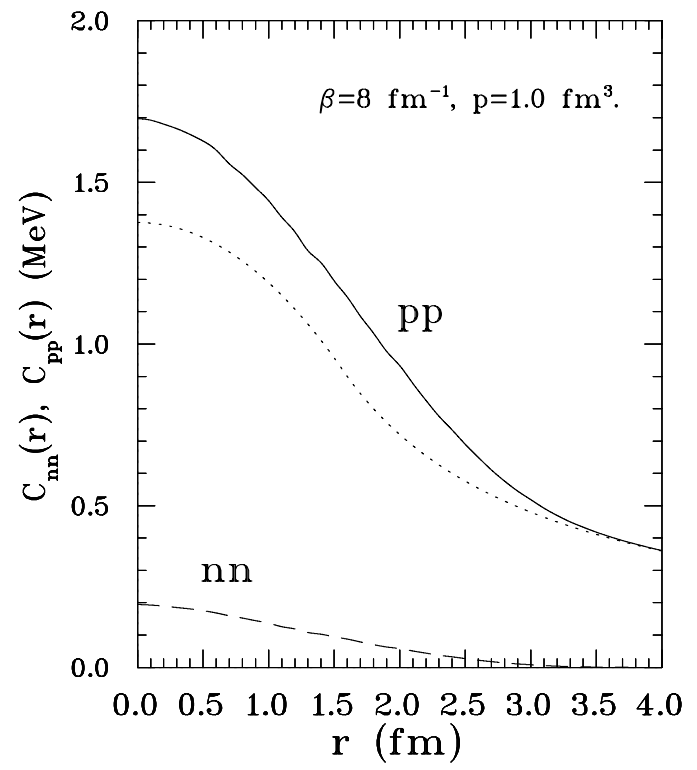

FIG. 9: Neutron-neutron and $p p$ potentials resulting from the calculations described in the text. The $p p$ case includes the normal Coulomb interaction as well as the hidden part. The dotted line shows the potential from a uniformly charged sphere of radius $1.56 \mathrm{fm}, V_{C}(r)$. In order to find the CC part the differences in these curves is taken.

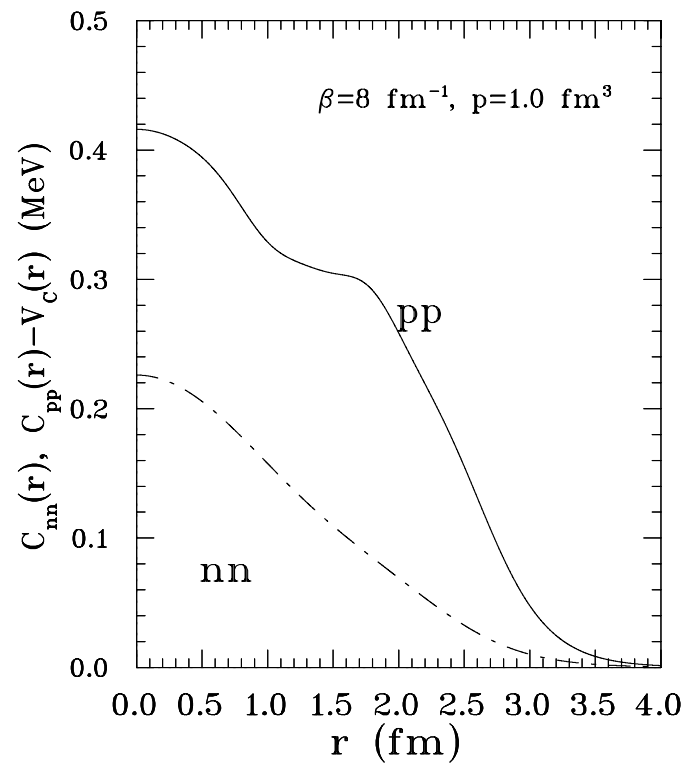

FIG. 10: Crypto-Coulomb potentials with the potential from a uniformly charged sphere of radius 1.56 fm subtracted from the $p p$ interior potential.

\section{C. ${ }^{3} \mathrm{He}^{3} \mathrm{H}$ mass difference}

Several cases have been calculated with our variational wave function introduced in Ref. [145].

In order to calculate with a realistic wave function we use a trial wave function which results from a variational calculation for ${ }^{3} \mathrm{H}$. Since the true wave function must be translationally invariant it can depend only on the relative coordinates, $\mathbf{r}_{i j}=\mathbf{r}_{i}-\mathbf{r}_{j}$.

We choose the basic form

$$
\psi\left(\mathbf{r}_{1}, \mathbf{r}_{2}, \mathbf{r}_{3}\right)=f\left(r_{12}\right) f\left(r_{13}\right) f\left(r_{23}\right)
$$

where $r_{i j}=\left|\mathbf{r}_{i j}\right|$ and the function, $f(r)$ is arbitrary at this point. It will be chosen with a number of parameters to be selected to minimize the energy. 


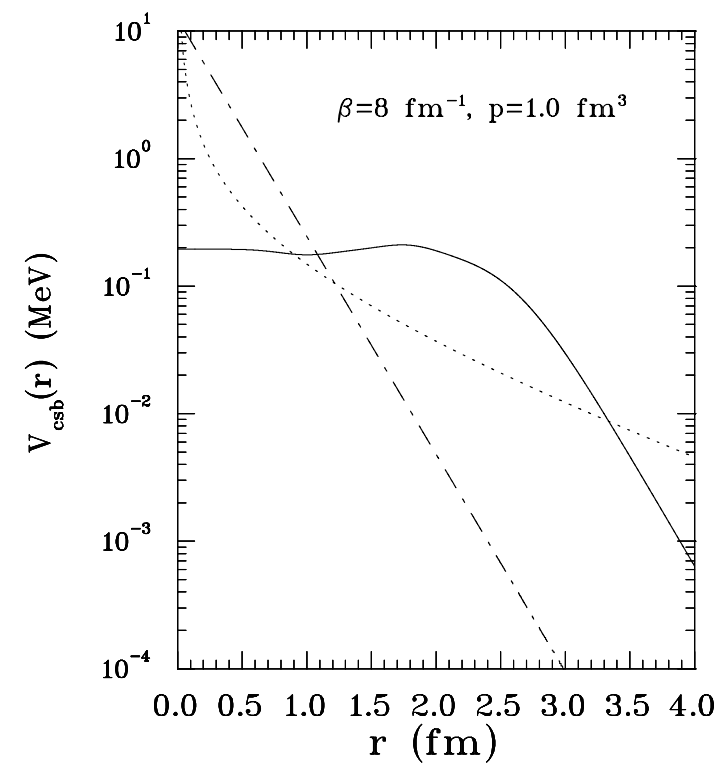

FIG. 11: CC charge symmetry breaking potential calculated by subtracting the uniformly charged potential and the $n n$ interior Coulomb potential from the $p p$ interior potential (solid line). The dotted line shows the shape of a one-pion-exchange potential with arbitrary normalization. The dash-dot line shows the first term of the $\rho$ - $\omega$ CSB potential (Equation 8 of ref. [80] with their parameter $\beta=0$ ).

$\begin{array}{ccccc} & \beta=4 \mathrm{fm}^{-1} & \beta=8 \mathrm{fm}^{-1} & \beta=12 \mathrm{fm}^{-1} & \text { Experimental value } \\ \mathrm{E}_{3} \mathrm{H}(\mathrm{MeV})(\text { With crypto-Coulomb) } & 8.5034 & 8.4966 & 8.4999 & 8.4818 \\ \mathrm{E}_{3_{\mathrm{H}}}(\mathrm{MeV}) \text { (Pure Isospin) } & 8.5475 & 8.5452 & 8.5490 & \\ \text { Difference (keV) } & 44.1 & 48.6 & 49.1 & \\ \mathrm{E}_{3 \mathrm{He}}(\mathrm{MeV})(\text { With crypto-Coulomb) } & 7.7869 & 7.7760 & 7.7770 & 7.7181 \\ \mathrm{E}_{3} \mathrm{He}(\mathrm{MeV})(\text { Charged sphere) } & 7.8810 & 7.8787 & 7.8825 & \\ \text { Difference (keV) } & 99.4 & 102.7 & 105.5 & \\ \text { Difference between }{ }^{3} \mathrm{He} \text { and }{ }^{3} \mathrm{H}(\mathrm{keV}) & 716.5 & 720.1 & 720.6 & \\ \text { Including } 46 \mathrm{keV} & 762.5 & 766.1 & 766.6 & 763.8\end{array}$

TABLE III: Coulomb differences in the 3 -nucleon system. The $46 \mathrm{keV}$ in the last line comes from the estimate of several small effects by Wu, Ishikawa and Sasakawa [84] and Friar, Gibson and Payne [85].

We originally took for the form of $f$

$$
f(r)=\left(1-e^{-c^{\prime} r}\right) \frac{e^{-a r}}{b+r}
$$

the first factor vanishes at the origin and provides the effect of a (very mild) repulsive correlation. The rest of the function has the proper asymptotic form. For the relatively long-range isospin breaking potentials that we have used before, the very short-range region was not crucial but with the full NN potential, with its strong repulsion, we need to model the short-range exclusion region of the factors. To this end we include an additional factor in $f(r)$ which is

$$
e^{-\alpha_{n} / r^{n}}
$$

where both $n$ and $\alpha_{n}$ can be treated as variational parameters.

The $n p$ interaction was modified so as to obtain approximately the correct binding energy for ${ }^{3} \mathrm{H}$ by decreasing the repulsive core by $38 \%$. Since we have set the parameters of the wave function to give the correct three-nucleon r.m.s. radius (we deem the range over which the potential is tested to be important) this choice of an $n p$ potential to give the correct binding energy is only cosmetic. The mass difference in the three-nucleon system could be obtained directly by the expectation value of the breaking CC potentials. 
See table IIIfor a summary of the results. The difference in energy between the conventional Coulomb and the crypto-Coulomb for ${ }^{3} \mathrm{He}$ is about $100 \mathrm{keV}$. Of course the Triton also has an effect from the $n n$ repulsion which, overall gives about $50 \mathrm{keV}$ less binding with the $\mathrm{CC}$ so the difference beyond the conventional Coulomb is near $50 \mathrm{keV}$ which is about what Wu, Ishikawa and Sasakawa [84] and Friar, Gibson and Payne [85] find is needed after a number of small effects have been taken into account.

\section{EXTENSION TO THE STRANGE SECTOR}

Similar considerations can be applied to the $\Lambda n$ and $\Lambda p$ systems. In the diquark picture the $\Lambda$ consists of quarks $u d$ coupled to $I=0$ with charge $+1 / 3$ and the strange quark with charge $-1 / 3$. Thus it is electrically the same as the neutron. If we were to assume that the masses and the interactions among the quarks were all the same, $\mathrm{SU}(3)$, then we could directly use the $n n$ and $n p$ crypto-Coulomb potentials obtained previously.

\begin{tabular}{|c|c|c|c|c|c|}
\hline case & $\begin{array}{c}\Lambda p a \\
\mathrm{fm}\end{array}$ & $\begin{array}{c}\Lambda n a \\
\mathrm{fm}\end{array}$ & $\begin{array}{c}\Lambda p r_{0} \\
\mathrm{fm}\end{array}$ & $\begin{array}{c}\Lambda n r_{0} \\
\mathrm{fm}\end{array}$ & $\% \operatorname{diff} a$ \\
\hline$O P E=0$ & -4.17 & -3.89 & 2.88 & 2.91 & 6.7 \\
\hline$C_{1} \times 0.9535$ & -2.51 & -2.36 & 3.46 & 3.51 & 6.0 \\
\hline$C_{2} \times 1.0785$ & -2.51 & -2.37 & 3.53 & 3.58 & 5.5 \\
\hline$V \times 0.882$ & -2.51 & -2.37 & 3.33 & 3.37 & 5.5 \\
\hline NSC97f & -2.51 & -2.68 & 3.03 & 3.07 & -6.8 \\
\hline $\mathrm{ESC} 08 \mathrm{c}$ & -2.46 & -2.62 & 3.14 & 3.17 & -6.5 \\
\hline NSC89 & -2.73 & -2.86 & 2.87 & 2.91 & -4.7 \\
\hline
\end{tabular}

TABLE IV: SU(3) predictions for singlet $\Lambda N$ scattering parameters. The calculations were made with NN potentials which give $a_{n p}=-23.54 \mathrm{fm}, a_{n n}=-18.09 \mathrm{fm}, r_{0}(n p)=2.69 \mathrm{fm}$ and $r_{0}(n n)=2.82 \mathrm{fm}$. The last two lines are from fits by the Nijmegen group. NSC97f is from Rijken et al. [146], ESC08c is from Nagels et al. [147], and NSC89 is from Maessen et al. [148]. Our calculations are for $p=1.0 \mathrm{fm}^{3}$ and $\beta=8.0 \mathrm{fm}^{-1}$.

Table IV shows the results of several variations. The first line shows the result of removing the onepion-exchange potential. We see already that the right order of magnitude is obtained. Then, by reducing the strength of the remaining potential in three different ways one can match the NSC97f $\Lambda p$ result of Rijken et al. [146].

Rijken et al. [146] give six fits to hyperon-nucleon scattering data of equivalent quality labeled a-f. The solution $f$ is emphasized in the paper and their G-matrix calculation of hyperonic systems also favors it. Miyagawa et al. 149] give limits on the singlet and triplet scattering and only the $f$ solution falls within these limits. In Ref. [150] the singlet and triplet scattering lengths were extracted from the results of a feasibility experiment of radiative Kaon capture on deuterium. While the limits on the singlet scattering length are too large $(-0.15 \rightarrow-5.0 \mathrm{fm})$ for a comparison to be useful, the triplet determination $(-1.3 \rightarrow-2.65 \mathrm{fm})$ does provide some constraint and is in agreement with the triplet version (not shown) of all of the potentials corresponding to the scattering parameters shown in the last three lines of Table IV. The principal isospin breaking mechanism used in the potentials of Rijken et al. [146] (and others) is $\Lambda \Sigma$ mixing, first introduced by Dalitz and von Hippel (DvH) [151].

The potential NSC89 [148] deserves further discussion. It has a larger $\Lambda \Sigma$ mixing in the few-baryon system and so lends itself to the understanding of the anomalous $\pi^{+}$weak decay of ${ }_{\Lambda}^{4} \mathrm{He}$ [152]. However, perhaps partly due to the fact that it gives twice the excitation energy of the $1^{+}$state in ${ }_{\Lambda}^{4} \mathrm{He}$ than that observed, it seems to have been rejected by the community and it has been said that "NSC89 is definitely not a realistic YN potential for use in hypernuclei [153]."

The potential ESC08c comes [147, 154, 155] from a set of potentials which use the forbidden state concept to implement the Pauli blocking at short distances and includes explicit two-pion exchange.

We see that Nijmegen breaking potentials are more attractive for the $\Lambda n$ interaction than for the $\Lambda p$ interaction in the singlet channel. The opposite is true for the triplet channel. This behavior is expected for the $\Lambda \Sigma$ mixing mechanism for the breaking as by the formula given by Gal [156]. Gal gives [136, 156] a simple relation of the breaking potential to the strong interaction potential causing the transition $\Lambda \rightarrow \Sigma$. The interaction has a major component of one-pion exchange (OPE). 
The OPE potential consists of a central (spin-spin) and a tensor term. If only the spin-spin term were present, the singlet to triplet ratio of the breaking potentials would be $-3: 1$. The tensor part contributes (only in the triplet state) and alters this ratio. For the Nijmegen triplet scattering lengths (not shown) we estimate that the ratio of the corresponding breaking potentials is about -1:1.

The percentage breaking observed for the triplet state is about the same as the singlet but in the same direction as the $\mathrm{CC}$ effect. Assuming that both $\mathrm{DvH}$ and $\mathrm{CC}$ potentials contribute, we would expect that they tend to cancel in the singlet and add constructively in the triplet. This would lead to a large breaking $(\approx 12 \%)$ in the triplet state, a fortunate circumstance since the $\Lambda n$ triplet appears $[150$ ] to be easier to extract from data than the singlet.

As in the non-strange sector, there are tests available in mirror hypernuclei, the most common being the ground $\left(0^{+}\right)$and first excited states $\left(1^{+}\right)$of ${ }_{\Lambda}^{4} \mathrm{H}$ and ${ }_{\Lambda}^{4} \mathrm{He}$.

The experimental data come from several sources which are enumerated in Yamamoto et al. [157]. The ground-state binding energies were measured with an emulsion technique by Jurić et al. [158] $B^{0+}\left({ }_{\Lambda}^{4} H e\right)=2.39 \pm 0.03 \mathrm{MeV}$ and $B^{0+}\left({ }_{\Lambda}^{4} H\right)=2.04 \pm 0.04 \mathrm{MeV}$. A modern measurement was presented by Esser et al. [159], $B^{0+}\left({ }_{\Lambda}^{4} H\right)=2.12 \pm 0.09 \mathrm{MeV}$. The binding energy of the $1^{+}$excited state is obtained by adding the energy difference obtained from the measured energy of the $\gamma$ from the transition. Yamamoto et al. [157] (aside from their own measurement of the transition energy in ${ }_{\Lambda}^{4} \mathrm{He}=1.406 \pm 0.003$ ) obtained the $\gamma$ energy for ${ }_{\Lambda}^{4} \mathrm{H}=1.09 \pm 0.02 \mathrm{MeV}$ from the average of three experiments [160 162]. The breaking data are summarized in table V. Experimental column $a$ is calculated using the ground-state measurements of Jurić et al. [158] while column $b$ substitutes the measurement of Esser et al. [159] for $B^{0+}\left({ }_{\Lambda}^{4} \mathrm{H}\right)$. Column $c$ shows the breaking in the excitation energy from Yamamoto et al. [157].

Nogga et al. [163] addressed the challenges of choosing the "best" hyperon-nucleon interaction by investigating the set of potentials mentioned above [146]. They found that NSC97f under-binds ${ }_{\Lambda}^{3} \mathrm{H}$ (but by considerably less than the rest of the 97 set) while NSC89 slightly over-binds the three-baryon system. Mirror-nuclei tests are not available in this system.

For the difference in energy between the ground state and the $1^{+}$excited state in the four-body system they found that NSC97f gives the correct value although the absolute binding is too small. No potential was found to give the correct values for all observables.

They also studied the the contribution of the spin to the total angular momentum and found that the ground state is about $90 \% \mathrm{~S}=0$ and the first excited state is $96 \% \mathrm{~S}=1$. If one were to take the like-nucleon pair to be coupled to spin zero in a pure s-wave model, the $\Lambda$-odd nucleon pair would determine the spin of the total system. In this case the ground state would be pure $\Lambda-N$ singlet and the excited state pure triplet. The percentages found by Nogga et al. [163] are reasonably consistent with this simple picture.

Nogga et al. [163] also calculated the effect of the CSB on the difference in separation energies of the ${ }_{\Lambda}^{4} \mathrm{H}$ and ${ }_{\Lambda}^{4} \mathrm{He}$ systems. They found that NSC97e gives only $70 \mathrm{keV}$ of the experimental value of 270-350 $\mathrm{keV}$. On the other hand NSC89 gives essentially all that is needed.

We see that our results show a more attractive $\Lambda p$ than $\Lambda n$ potential reflected in the fact that the scattering length is more negative. This is in the direction needed since the separation energy of the $\Lambda-{ }^{3} \mathrm{He}$ system (2.39) Jurić et al. [158] (see also Esser et al. [159] is greater than that of the $\Lambda-{ }^{3} \mathrm{H}$ system from Jurić et al. [158] $(2.04 \mathrm{MeV})$ or Esser et al. [159] $(2.12 \mathrm{MeV})$. The singlet scattering lengths of Rijken et al. 146] show the opposite behavior. The crypto-Coulomb attraction in the $\Lambda p$ system and the repulsion in the $\Lambda n$ system can be expected to be about the same in the triplet as the singlet since their origin is the correlation of the odd quarks of different or same charge.

Since the resulting charge symmetry breaking potential

$$
C_{\Lambda N}(r) \approx C_{n p}(r)-C_{n n}(r)
$$

is of the order of 300-400 keV (see Fig. 12 and the difference of the separation energies is 270-350 $\mathrm{keV}$, it seems that one is bound to find a significant fraction of this difference from this source. From general considerations in the present model we see that CSB in the strange sector (by the measure of the separation energies) corresponds to CIB in the non-strange sector.

An important contribution to the understanding of CSB in the four-body system was recently reported by Gazda and Gal [136]. They present the results of no-core shell model calculations of ${ }_{\Lambda}^{4} \mathrm{He}$ and ${ }_{\Lambda}^{4} \mathrm{H}$ for the ground state $\left(0^{+}\right)$and first excited state $\left(1^{+}\right)$. They use a CSB interaction from the DvH [151] mechanism which has (largely) pion coupling to mix the isospin-zero $\Lambda$ with the isospin-one $\Sigma^{0}$. They use YN potentials from Polinder et al. [134] calculated with effective field theory to leading order and available for a range of values of cut-off mass. They find a strong dependence on the cut-off mass (see Table V). Restricting ourselves to the lower values of $\Lambda_{q}$ we see that the breaking in the ground state is compatible with the $70 \mathrm{keV}$ found by Nogga et al. [163], much smaller than the experimental value of 270-350 keV. 


\begin{tabular}{lcccc|ccc}
\hline$\Lambda_{g}(\mathrm{MeV})$ & 550 & 600 & 650 & 700 & $\operatorname{Exp}(\mathrm{a})$ & $\operatorname{Exp}(\mathrm{b})$ & $\operatorname{Exp}(\mathrm{c})$ \\
\hline$\Delta^{1+}=B^{1+}\left({ }_{\Lambda}^{4} H e\right)-B^{1+}\left({ }_{\Lambda}^{4} H\right)$ & -172 & -193 & -228 & -223 & $+30 \pm 50$ & $-50 \pm 90$ & \\
$\Delta^{0+}=B^{0+}\left({ }_{\Lambda}^{4} H e\right)-B^{0+}\left({ }_{\Lambda}^{4} H\right)$ & 30 & 136 & 244 & 294 & $350 \pm 50$ & $270 \pm 130$ & \\
$\Delta^{0+}-\Delta^{1+}$ & 202 & 329 & 492 & 517 & & & $316 \pm 20$ \\
\hline
\end{tabular}

TABLE V: Isospin breaking energy differences as a function of cut-off parameter taken from Gazda and Gal [136] (columns 2-5) compared with measurements (columns a-c). The experimental energy breaking difference between the ground and first excited state (column c) is taken directly from the measurements of the difference in the $\gamma$ energies [157]. See the text for references to the data.

The breaking in the difference of the energies is equivalent to the breaking in the directly observed $\gamma$ rays [157]. The breaking in the excited state is calculated [136] to be around $-200 \mathrm{keV}$ whereas the experimental value is around zero. A constant shift of the breaking in both states $\left(0^{+}\right.$and $\left.1^{+}\right)$of $\approx 200$ $\mathrm{keV}$ would give agreement with data. An energy shift of $200 \mathrm{keV}$ is about the result expected from the $\mathrm{CC}$ mechanism presented here since, from arguments given earlier, we expect little or no spin dependence for the CC mechanism.

Thus the mixing $(\mathrm{DvH})$ mechanism gives attraction for the ground state and repulsion for the excited state. This is perhaps understandable since the spin-spin coupling which is providing the mixing changes sign between singlet and triplet states in the usual manner.

It may be useful to discuss the possibly confusing result that the maximum breaking in the scattering lengths occurs in the triplet $\left(1^{+}\right)$state while the maximum breaking in the bound state is in the singlet $\left(0^{+}\right)$state. The CC effect is attractive for the $\Lambda p$ interaction and independent of spin so it increases the binding in ${ }_{\Lambda}^{4} \mathrm{He}$ (relative to ${ }_{\Lambda}^{4} \mathrm{H}$ ) in both states. It was pointed out by Gal [156] that the two like nucleons in the three-body core are paired to spin zero, hence do not interact with the mixing pion exchange leaving only the odd nucleon to cause the mixing. Since, with the $\mathrm{DvH}$ mechanism, the $\Lambda n$ breaking interaction is more attractive in the singlet state, its interaction with the single neutron in the nucleon core leads to an attractive interaction in the ${ }_{\Lambda}^{4} \mathrm{He}$ case and it adds to the CC potential constructively. Since the DvH potential changes sign for the triplet case, the two potentials have opposite signs, leading to a smaller breaking

It may be useful to use the breaking in the energy of the $\gamma$ between the two states as a constraint 136 since it has the smallest experimental uncertainty. Doing that, in Table V we would choose $\Lambda_{g}=600$ $\mathrm{MeV}$ to compare. Adding $200 \mathrm{keV}$ to the two bindings leads to rough agreement with the data.

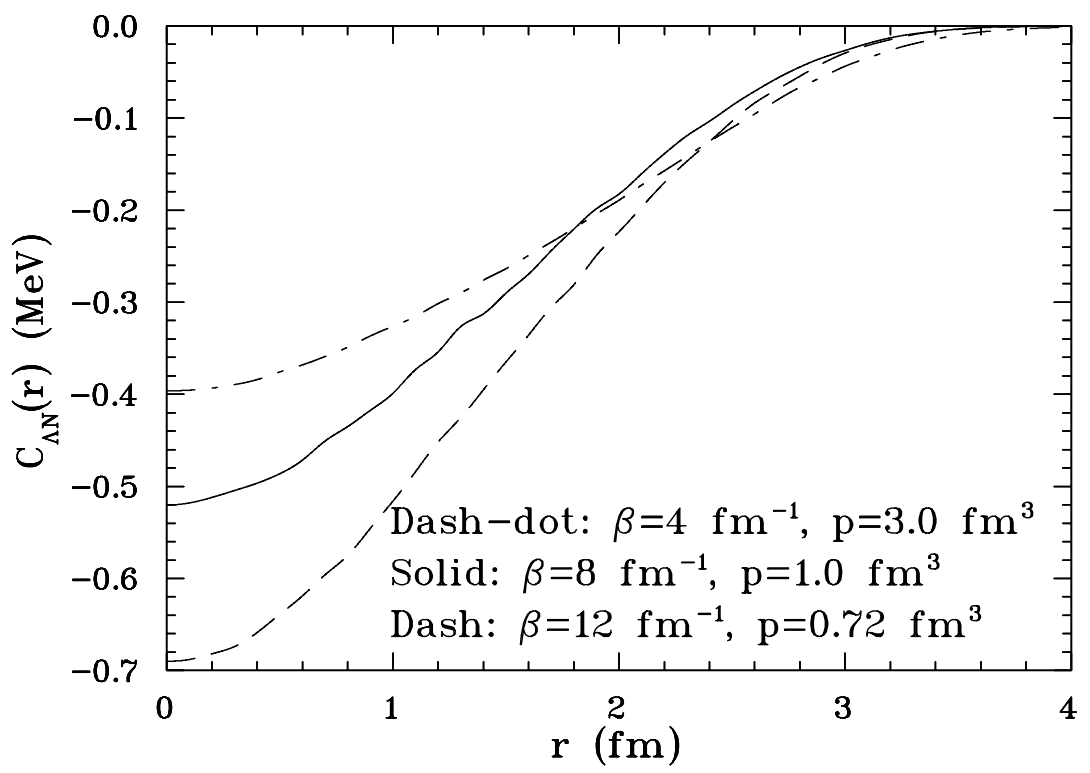

FIG. 12: Three examples of $\Lambda N$ breaking potentials from Eq. 25

There is one caveat in the comparison with the results of Gazda and Gal [136]. Their calculation was 
done with the fit to the leading order effective field theory [134]. Doing the same calculation with the next to leading order as input [135] leads to results inconsistent with data [136].

\section{DISCUSSION}

The general idea presented is that quark clustering will lead to isospin breaking through the Coulomb interaction. We have attempted to present this notion with a very simple illustrative model based solely on valence quarks. Since the breaking interaction arises from the Coulomb potential, it addresses CSB and CIB on an equal footing. Of course, the breaking due to the pion mass difference contributes to the CIB as well although we find that its influence is considerably reduced due to form factors.

If it seems to the reader that we are attacking the problem of the low energy $N N$ interaction by starting in the middle, it is because we are. Our object is to show that it is possible to establish a link between the quark rearrangement, which must occur in any complete theory of $N N$ scattering and isospin breaking. We have attempted to do so by considering a simple model, in which we included what we believe to be the basic physics of the system. Since only the quark density is needed to calculate the Coulomb energy many of the details of the wave function are unnecessary, allowing progress to be made with a minimum of assumptions.

The meson-nucleon form factor range plays a significant role in the understanding of CSB and CIB. The value of the regulating mass that we have adopted may appear small to some but we believe we have justified it with a number of references. It is interesting to note that, in the effective field theory approach, both in the non-strange [129 131] and strange sectors [134, 135] the minimum $\chi^{2}$ is obtained for values of $\Lambda_{g}$ around $500-600 \mathrm{MeV} / \mathrm{c}$, in general agreement with previous determinations of the cut-off cited in Section IV D and also compatible with the value derived from the size of the nucleon in the same section.

The value that we obtained for the magnitude of the "Coulomb corrected" $p p$ scattering length $(-16.2 \mathrm{fm})$ is smaller than the accepted one $(-17.3 \pm 0.4 \mathrm{fm})$ although the error on the latter is perhaps optimistic. Our value does not depend on the CC potential (removing it in the fit and the calculation of the "strong" calculation gives essentially the same value), nor does it depend on the ranges $\mu_{1}$ and $\mu_{2}$ in Eqs. (10[11) within limits. The difference may very well come from the fact that we fit a different data set than others. One sensitivity that was observed is to the short-range Coulomb. For example, the use of a point Coulomb potential would lead to a $p p$ scattering length of $-16.7 \mathrm{fm}$. In any case, the difference seems to represent a general shift since the $n n$ scattering length we obtain $\left(-18.0 \mathrm{fm}^{-1}\right)$ is also less negative than the measured value such that the breaking, often expressed as

$$
\left|a_{n n}\right|-\left|a_{p p}\right|
$$

is $1.8 \mathrm{fm}$ compared with $1.6 \pm 0.6 \mathrm{fm}[\underline{120}]$.

Up until this point we have said little about the spin of the diquark, it does not enter directly in the calculation. There is an implicit dependence, however. Since we assume that the odd quarks always couple to form the same correlation then, assuming an energy determined by the spin since the like quarks must couple to spin 1 the $u d$ quarks would need to be in a spin 1 state as well, the spin 0 and spin 1 diquarks would need to have the same interaction, or there could be some mixture of the two diquarks.

A number of papers have touched on this topic. Aside from those mentioned earlier, Close and Thomas 164 studied quark distributions with an axial vector diquark heavier than the scalar diquark. Mineo et al. 19] consider a mixture and conclude that the axial vector diquark should have a weight of 2-10\%. Cloët et al. 165. find the inclusion of the axial vector diquark important for the nucleon electromagnetic form factors and the quark flavor distribution. Nagata and Hosaka [166] find the axial vector diquark to be important for understanding the nucleon charge form factors. Finally, and perhaps most important, is the work of Cloët and Miller [63] (already mentioned in Section II) where the inclusion of the axial vector diquark leads to a possible understanding of the proton-spin puzzle. We see that recent work has indicated that the axial vector diquark may play a crucial role in the structure of the nucleon.

There would seem to be a fundamental difficulty with the assumption of only a scalar diquark for NN scattering. If the $u d$ quarks are assumed to form a scalar diquark then, in the $n n$ and $p p$ scattering the two "odd" quarks must form a spin one object in the symmetric quark model (the axial diquark). One need not assume any clustering for this argument. Hence, the two nucleon system, which must be in a singlet state, cannot be formed. Thus, nucleons built up from a pure scalar diquark cannot generate the low-energy S-wave scattering. If the $u d$ pair always is a combination of scalar and axial vector diquarks then a coupling to total spin zero is possible for all three $N N$ pairs.

We finish with some general comments: 
1) The CC model for isospin symmetry breaking depends only on the density of the quarks and not (directly at least) on their wave functions or spin. For this reason, obtaining the correct breaking is not a strong test of diquark models, although it provides a testing mechanism.

2) The non-zero-energy amplitude might also be calculated and may give information about the rearrangement reaction. This might come about through the study of the breaking in the effective range, $r_{0}$, for example. A better understanding of the $n D$ breakup reaction could provide crucial input in this regard.

3) Since models of the type we considered here are based only on the charges of the quarks, they are readily extensible to other sectors. The strange sector appears to be especially useful in this regard. Taking over results from the non-strange sector the CC concept is capable providing an understanding of the recent result of Gazda and Gal [136] of a state-independent contribution to the breaking of the order of 200-300 keV. Of course other models may give a similar prediction but we know of no other at the present time.

One of us (WRG) acknowledges several very valuable conversations with B. F. Gibson. WRG also acknowledges the hospitality of the Laboratoire de Physique Nucléaire et de Hautes Énergies (LPNHE) where part of this work was done.

\section{Appendix A: Analytic fits}

In each case the parameters $C_{1}$ and $C_{2}$ are those in Eq. 10, $M=2 \mathrm{fm}^{-1}$. For reference, the original singlet S-wave Reid [10] soft-core potential had $C_{1}=-1650.6 \mathrm{MeV}$ and $C_{2}=6484.2 \mathrm{MeV}$.

$$
\begin{gathered}
\beta=12 \mathrm{fm}^{-1}, \mathrm{p}=0.58 \mathrm{fm}^{3} \\
V_{C S B}(r)=C_{p p}(r)-V_{C}(r)-C_{n n}(r)=\frac{0.35}{\left(1+e^{(r-.5) / 1.2}\right)}+0.07 e^{-(r-1.75)^{2} / 0.12} \\
-0.019 r e^{-10(r-3.5)^{2}}+0.04 e^{-(r-1.8)^{2} / 0.6} \\
C_{n n}(r)=0.21 e^{-r^{2} / 2.6}+0.011 r e^{-(r-1.8)^{2} / 0.6}
\end{gathered}
$$

Parameters for $V_{0}(r): C_{1}=1757.87 \mathrm{MeV}, C_{2}=7075.00 \mathrm{MeV}$

$$
\begin{gathered}
\beta=8 \mathrm{fm}^{-1}, \mathrm{p}=1.0 \mathrm{fm}^{3} \\
V_{C S B}(r)=C_{p p}(r)-V_{C}(r)-C_{n n}(r)=\frac{0.19}{\left(1+e^{4(r-2.65)}\right)}+0.023 e^{-12.5(r-1.78)^{2}}-0.019 r e^{-10(r-0.95)^{2}} \\
C_{n n}(r)=0.226 e^{-r^{2} / 2.6}+0.011 r e^{-(r-1.8)^{2} / 0.6}
\end{gathered}
$$

Parameters for $V_{0}(r): C_{1}=1792.88 \mathrm{MeV}, C_{2}=7150.00 \mathrm{MeV}$

$$
\begin{gathered}
\beta=4 \mathrm{fm}^{-1} \mathrm{p}=3.0 \mathrm{fm}^{3} \\
V_{C S B}(r)=C_{p p}(r)-V_{C}(r)-C_{n n}(r)=0.175 e^{-(r-1.93)^{2} / 0.75}-0.03 e^{-1.2 r} \\
C_{n n}(r)=0.17 e^{-(r / 1.2)^{2}}+0.05 e^{(r-1.8)^{2}}
\end{gathered}
$$

Parameters for $V_{0}(r): C_{1}=1809.57 \mathrm{MeV}, C_{2}=7400.00 \mathrm{MeV}$

[1] M. Lacombe, B. Loiseau, J. M. Richard, R. Vinh Mau, J. Conté, P. Pires and R. de Tourreil, Phys. Rev. C 21, $861(1980)$ 
[2] R. Vinh Mau, C. Semay, B. Loiseau and M. Lacombe, Phys. Rev. Lett. 67, 1392 (1991)

[3] S. Takeuchi, K. Shimizu and K. Yazaki, Nucl. Phys. A504, 777 (1989)

[4] M. Oka and K. Yazaki, Phys. Lett. B90, 41 (1980)

[5] Y. Yamauchi, R. Yamamoto and M. Wakamatsu, Nucl. Phys. A443, 628 (1985)

[6] M. C. M. Rentmeester, R. G. E. Timmermans, J. L. Friar, and J. J. de Swart, Phys. Rev. Lett. 824992 (1999)

[7] J.C.R. Bloch, C.D. Roberts, S.M. Schmidt, A. Bender, and M.R. Frank, arXiv:nucl-th/9907120v1 (1999)

[8] J. Carlson, J.B. Kogut and V.R. Pandharipande, Phys. Rev. D 27, 233 (1983)

[9] J. Carlson, J.B. Kogut and V.R. Pandharipande, Phys. Rev. D 28, 2807 (1983)

[10] R. V. Reid, Annals of Phys. 50, 411 (1968)

[11] M. Gell-mann, Phys. Lett. 8, 214 (1964)

[12] M. Ida and R. Kobayashi, Prog. Theor. Phys.36, 846 (1966)

[13] D. B. Lichtenberg and L. J. Tassie, Phys. Rev. 1551601 (1967)

[14] S. Ono, Phys. Rev. D 10, 3124 (1974); Nuovo Cimento Lett. 8, 378 (1973); Prog. of Theo. Phys. 50, 589 (1973); Prog. of Theo. Phys. 49, 573 (1973); Prog. of Theo. Phys. 48, 964 (1972)

[15] D. B. Lichtenberg, W. Namgung, E. Predazzi, and J. G. Wills, Phys. Rev. Lett. 48, 1653 (1982)

[16] D. B. Lichtenberg, E. Predazzi, D. H. Weingarten, and J. G. Wills, Phys. Rev. D 18, 2569 (1978)

[17] L. F. Abbott, E. L. Berger, R. Blanckenblecler, and G. L. Kane, Phys. Lett. B88, 157 (1979)

[18] J. F. Gunion, Phys. Rev. D 10, 342 (1974)

[19] H. Mineo, W. Bentz, N. Ishii, and K. Yazaki, Nucl. Phys. A703, 785 (2002)

[20] M. Anselmino, E. Predazzi, S. Ekelin, S. Fredriksson and D. B. Lichtenberg, Rev. Mod. Phys. 65, 1199 (1993)

[21] C. Alexandrou, Ph. de Forcrand and B. Lucini, Phys. Rev. Lett. 97, 222002 (2006)

[22] M. Hess, F. Karsch, E. Laermann and I. Wetzorke, Phys. Rev. D 58, 111502 (1998)

[23] R. Babich, N. Garron, C. Hoelbling, J. Howard, L. Lellouch and C. Rebbi, Phys. Rev. D 76, 074021 (2007)

[24] C. Alexandrou, Ph. de Forcrand and B. Luicini, PoS LAT2005,053 (2005); arXiv: hep-lat/0509113

[25] J. Green, M. Engelhardt, J. Negele and P. Varilly, AIP Conf. Proc. 1441, 172 (2012) and PoS LATTICE 2010, (2010); arXiv 1012.2353

[26] C. Weiss, A. Buck, R. Alkofer and H. Reinhardt, Phys. Lett. B312, 6 (1993)

[27] N. Ishii, W. Bentz and K. Yazaki, Phys. Lett. B318, 26 (1993)

[28] V. Keiner, Phys. Rev. C 54, 3232 (1996)

[29] X.-H. Guo, A. W. Thomas and A. G. Williams, Phys. Rev. D 59, 116007 (1999)

[30] M. H. Weng, X.-H. Guo and A. W. Thomas, Phys. Rev. D 83, 056006 (2011)

[31] I. C. Cloët, W. Bentz and A. W. Thomas, Phys. Rev. C 90, 045202 (2014) and references therein

[32] E. Santopinto and J. Ferretti, Phys. Rev. C 92, 025202 (2015)

[33] D. Ebert, R. N. Faustov and V. O. Galkin, Phys. Rev. D 84, 014025 (2011)

[34] D. M. Brink and F. Stancu, Phys. Rev. D 57, 6778 (1998)

[35] L. Maiani, V. Riquer, F. Piccinini, and A. D. Polosa Phys. Rev. D 72, 031502(R) (2005)

[36] L. Maiani, F. Piccinini, A. D. Polosa and V. Riquer, Phys. Rev. D 71, 014028 (2005)

[37] L. Maiani, A. D. Polosa and V. Riquer, New Journal of Physics 10, 073004 (2008)

[38] L. Maiani, A. D. Polosa and V. Riquer, Phys. Rev. Lett. 99, 182003 (2007)

[39] I. Bigi, L. Maiani, F. Piccinini, A. D. Polosa and V. Riquer, Phys. Rev. D 72, 114016 (2005)

[40] S. Dubnicka, A. Z. Dubnickova, M. A. Ivanov, J. G. Körner, Phys. Rev. D 81, 114007 (2010)

[41] K. Terasaki, Prog. Theo. Phys. 118, 821 (2007), arXiv:0706.3944 [hep-ph]

[42] K. Terasaki, Prog.Theor.Phys. 1221285 (2010), arXiv:0904.3368 [hep-ph]

[43] Y. Yang, C. Deng, J. Ping and T. Goldman, Phys. Rev. D 80, 114023 (2009)

[44] R. T. Kleiv, T. G. Steele, A. Zhang and I. Blokland, Phys. Rev. D 87, 125018 (2013)

[45] F. Piccinini, Nucl. Phys. A782, 362 (2007)

[46] D. Ebert, R. N. Faustov, V. O. Galkin and W. Lucha, Phys. Rev. D 76, 114015 (2007)

[47] D. Ebert, R. N. Faustov and V. O. Galkin, arXiv:0705.2957 v2 [hep-ph] (2007)

[48] D. Ebert, R. N. Faustov and V. O. Galkin, Phys. Lett. B634, 214 (2006)

[49] J. Vijande, A. Valcarce and J.-M. Richard, Phys. Rev. D 87, 034040 (2013)

[50] M. Padmanath, C. B. Lang and S. Prelovsek, Phys. Rev. D 92, 034501 (2015)

[51] B. Aubert et al. (BaBar Collaboration), Phys. Rev. D 73, 011101 (2006)

[52] S.-K. Choi et al. (Belle Collaboration), Phys. Rev. Lett. 100, 142001 (2008)

[53] R. Aaij et al. (LHCb Collaboration), Phys. Rev. Lett. 112, 222002 (2014)

[54] J. Weinstein and N. Isgur, Phys. Rev. D 27, 588 (1983)

[55] J. Weinstein and N. Isgur, Phys. Rev. D 41, 2236 (1990)

[56] C. Deng, J. Ping, H. Wang, P. Zhou and F. Wang, Phys. Rev. D 86, 114035 (2012)

[57] D. Ebert, R. N. Faustov and V. O. Galkin, Eur. Phys. J. C 60, 275 (2009)

[58] D. M. Brink and F. Stancu, Phys. Rev. D 49, 4665 (1994)

[59] L. Maiani, F. Piccinini, A. D. Polosa and V. Riquer, Phys. Rev. Lett. 93, 212002 (2004)

[60] A. Zhang, T. Huang and T. G. Steele, Phys. Rev. D 76, 036004 (2007)

[61] F. Myhrer and A. W. Thomas, Phys.Lett. B663, 302 (2008)

[62] A. W. Thomas, Phys. Rev. Lett. 101, 102003 (2008) 
[63] I. C. Cloët and G. A. Miller, Phys. Rev. C 86, 015208 (2012)

[64] W. Bentz and A. W. Thomas, Nucl. Phys. A696, 138 (2001)

[65] T. Horikawa and W. Bentz, Nucl. Phys. A762, 102 (2005)

[66] W. Bentz, T. Horikawa, N. Ishii, and A. W. Thomas, Nucl. Phys. A720, 95 (2003)

[67] F. Lenz et al., Ann. of Phys. (N.Y.) 170, 65 (1986)

[68] P. U. Sauer, Phys. Rev. C 11, 1786 (1974)

[69] C. T. H. Davies, et al., HPQCD, Phys. Rev. Lett. 104, 132002 (2010)

[70] R. P. Bickerstaff and A.W. Thomas, J. Phys. G 15, 1523 (1989)

[71] S. Borsanyi et al., Science 347, 1452 (2015)

[72] J. Gasser and H. Leutwyler, Phys. Rep. 87, 77 (1982)

[73] E. R. Henley and G. A. Miller, Mesons in Nuclei, Eds M. Rho, D. Wilkinson, p 405, North Holland (1979)

[74] G. A. Miller, B. M. K. Nefkens and I. Slaus, Phys. Reports 194, 1 (1990)

[75] G. A. Miller. A. K. Opper and E. J. Stephenson, Annu. Rev. Nuc. Part. Sci. 56, 253 (2006)

[76] A. Gardestig, J., Phys./ G : Nucl. Part. Phys. 36, 053001 (2009)

[77] K. Okamoto, Phys. Lett. 11, 150 (1964); K. Okamoto and C. Pask, Ann. of Phys. 68, 18 (1971)

[78] J. A. Nolen, Jr., J. P. Schiffer, Ann. Rev. Nucl. Sci. 19, 471 (1964)

[79] P. G. Blunden and M. J. Iqbal, Phys. Lett. B198, 14 (1987)

[80] S. A. Coon and R. C. Barrett, Phys. Rev. C 36, 2189 (1987); P. C. McNamee, M. D. Scadron and S. A. Coon, Nucl. Phys. A249, 483 (1975)

[81] H. Sato, Nucl. Phys. A269, 378 (1976)

[82] D. Vautherin and D. M. Brink, Phys. Rev. C 5, 626 (1972)

[83] J. W. Negele and D. Vautherin, Phys. Rev. C 5, 1472 (1972)

[84] Y. Wu, S. Ishikawa, and T. Sasakawa, Phys. Rev. Lett. 64, 1875 (1990)

[85] J. L. Friar, B. F. Gibson and G. L. Payne, Phys. Rev. C 42, 1211 (1990); Phys. Rev. C 35, 1502 (1987)

[86] G. L. Squires and A. T. Stewart, Proc. Royal Soc., Vol. 230, 19 (1955)

[87] A. T. Stewart and G. L. Squires, Phys. Rev. 90, 1125 (1953)

[88] I. Koester and W. Nistler, Z. Physik A272, 189 (1975)

[89] J. R. Bergervoet, P. C. van Campen, W. A. van der Sanden and J. J. de Swart, Phys. Rev. C 38, 15 (1988)

[90] B. Gabioud et al., Phys. Rev. Lett. 42, 1508 (1979)

[91] B. Gabioud et al., Phys. Lett B103, 9 (1981)

[92] B. Gabioud et al., Nucl. Phys A420, 496 (1984)

[93] O. Schori et al., Phys. Rev. C 35, 2252 (1987)

[94] C. R. Howell et al., Physics Letters B444, 252 (1998)

[95] Q. Chen et al., Phys. Rev. C 77, 054002 (2008)

[96] W. R. Gibbs, B. F. Gibson and G. J. Stephenson, Jr., Phys. Rev. C 11, 90 (1974)

[97] V. Huhn et al., Phys. Rev. Lett. 85, 1190 (2000)

[98] V. Huhn et al., Phys. Rev. C 63, 014003 (2000)

[99] C. R. Howell arXiv:0805.1177 1 [nucl-ex] (2008)

[100] D. E. Gonzalez Trotter et al., Phys. Rev. Lett. 83, 3788 (1999)

[101] D. E. Gonzalez Trotter et al., Phys. Rev. C 73, 034001 (2006)

[102] S. Shirato, K. Saitoh, N. Koori and R. T. Cahill, Nucl. Phys. A215, 277 (1973)

[103] E. M. Henley and I. K. Morrison, Phys. Rev. 141, 1489 (1966)

[104] S. A. Coon and M. D. Scadron, Phys. Rev. C 26, 2402 (1982)

[105] C. Y. Cheung and R. Machleidt, Phys. Rev. C 34, 1181 (1986)

[106] T. E. O. Ericson and G. A. Miller, Phys. Lett. B132, 32 (1983)

[107] J. L. Friar and U. van Kolck, Phys. Rev. C 60, 034006 (1999)

[108] G. Q. Li and R. Machleidt, Phys. Rev. C 58, 3153 (1998)

[109] T. Goldman, J. A. Henderson and A. W. Thomas, Few Body Systems 12, 123 (1992)

[110] J. Piekarewicz and A. G. Williams, Phys. Rev. C 47, R2462 (1993)

[111] T. Hatsuda, E. M. Henley, Th. Meissner and G. Krein, Phys. Rev. C 49, 452 (1994)

[112] G. Krein, A. W. Thomas and A. G. Williams, Phys. Lett. B317, 293 (1993)

[113] K. Maltman, Phys. Lett. B313, 203 (1993)

[114] H. B. O'Connell, B. C. Pearce, A. W. Thomas, and A. G. Williams, Phys. Lett. B335, 1 (1994)

[115] H. B. O'Connell, B. C. Pearce, A. W. Thomas, and A. G. Williams, Phys. Lett. B354, 14 (1995)

[116] K. L. Mitchell, P. C. Tandy, C. D. Roberts and R. T. Cahill, Phys. Lett. B335, 282 (1994)

[117] S. A. Coon, B. H. J. McKellar, and A. A. Rawlinson, Intersections of Particle and Nuclear Physics, Big Sky, MT, AIP conf Proc. Ed. T. W. Donnelly Vol. 412, 368 (1997)

[118] T. D. Cohen and G. A. Miller, Phys. Rev. C 52, 3428 (1995)

[119] S. A. Coon and J. A. Niskanen, Phys. Rev. C 53, 1154 (1996)

[120] R. Machleidt and H. Müther, Phys. Rev. C 63, 034005 (2001)

[121] W. M. Layson Nuovo Cimento 20, 1207 (1961)

[122] R. Landau and F. Tabakin, Phys. Rev. D 5, 2746 (1972)

[123] C. B. Dover, D. J. Ernst, R. A. Friedenberg, and R. M. Thaler, Phys. Rev. Lett. 33, 728 (1974)

[124] J. T. Londergan, K. W. McVoy and E. J. Moniz, Annals of Phys. 86, 147 (1974)

[125] D. J. Ernst and M. B. Johnson, Phys. Rev. C 17, 247 (1978) 
[126] U. T. Yakhshiev, A. W. Thomas and F. C. Khanna, Phys. Rev. C 68, 048201 (2003)

[127] V. I. Kukulin, I. T. Obukhovsky, V. N. Pomerantsev, and A. Faessler, Phys. of At. Nucl., 641667 (2001)

[128] E. Marji et al., Phys. Rev. C 88, 054002 (2013)

[129] D. R. Entem and R. Machleidt, Phys. Rev. C 68, 041001 (2003)

[130] F. Sammarruca, L. Coraggio, J. W. Holt, N. Itaco, R. Machleidt, L. E. Marcucci, Phys. Rev. C 91, 054311 (2015)

[131] F. Sammarruca, R. Machleidt and N. Kaiser, Phys. Rev. C 92, 054327 (2015)

[132] E. Epelbaum, W. Glöckle and U. Meissner, arXiv:nucl-th/0405048v2 (2004)

[133] E. Epelbaum and U. Meissner, arXiv:nucl-th/0609037v2 (2013)

[134] H. Polinder, J. Haidenbauer U.-G. Meissner, Nucl. Phys. 779, 244 (2006)

[135] J. Haidenbauer, S. Petschauer, N. Kaiser, U.-G. Meissner, A. Nogga and W. Weise, Nucl. Phys. A915, 24 (2013)

[136] D. Gazda and A. Gal, Phys. Rev. Lett. 116, 122501 (2016)

[137] M. Rho, A.I.P. Conf. Proc., Vol. 33, 146 (1976)

[138] R. M. Woloshyn, Phys. Rev. Lett. 36, 220 (1976)

[139] A. W. Thomas and K. Holinde, Phys. Rev. Lett. 63, 2025 (1989); Phys. Rev. C 42, R1195 (1990)

[140] H. R. Rubinstein, /prl 17, 41 (1966)

[141] A. Gal and F. Scheck, Nucl. Phys, B2, 110 (1967)

[142] D. B. Lichtenberg, Phys. Rev. D 14, 1412 (1976)

[143] L. Heller, Phys. Rev. 120, 627 (1960)

[144] A. K. Sisodiya, R. S. Kaushal, D. Parashar, and V. S. Bhasin, J. Phys. G: Nucl. Part. Phys. 32, 1193 (2006)

[145] W. R. Gibbs and J. P. Dedonder, Phys. Rev. C 86, 024604 (2012)

[146] Th. A. Rijken, V. G. J. Stoks and Y. Yamamoto, Phys. Rev. C 59, 21 (1999)

[147] M. M. Nagels, Th. A. Rijken, and Y. Yamamoto, arXiv:1501.06636v1[Nucl-th]

[148] P. M. M. Maessen, Th. A. Rijken and J. J. de Swart, Phys. Rev. C 402226 (1989)

[149] K. Miyagawa, H. Kamada, W. Glöckle, H. Yamamura, T. Mart and C. Bennhold, in Proceedings of the First Asia-Pacific Few-Body Conference, Japan, Few-Body Systems Suppl. 12, 324 (1999)

[150] W. R. Gibbs, S. A. Coon, H. K. Han and B. F. Gibson, Phys. Rev. C 61, 064003 (2000)

[151] R. H. Dalitz and F. von Hippel, Phys. Lett. 10, 153 (1964)

[152] B. F. Gibson and R. G. E. Timmermans.Nucl. Phys. A628 417 (1998)

[153] A. Gal, E. V. Hungerford, and D. J. Millener, arXiv:1605.00557v1 [nucl-th] in press Rev. Mod. Phys.

[154] Y. Yamamoto, E. Hiyama and Th. A. Rijken, Nucl. Phys. A835, 350 (2010)

[155] Th. A. Rijken, M. M. Nagels and Y. Yamamoto, Nucl. Phys. A835, 160 (2010)

[156] A. Gal, Phys. Lett. B 744, 352 (2015)

[157] T. O. Yamamoto et al. (J-PARC E13-1st Collaboration), Phys. Rev. Lett. 115, 222501 (2015)

[158] M. Jurić et al., Nucl. Phys. B52, 1 (1973)

[159] A. Esser et al., Phys. Rev. Lett. 114, 232501 (2015)

[160] M. Bedjidian et al., Phys, Lett. B 62, 467 (1976)

[161] M. Bedjidian et al., Phys, Lett. B 83, 252 (1979)

[162] A. Kawachi, Doctoral Thesis, University of Tokyo (1997), unpublished

[163] A. Nogga, H. Kamada and W. Glöckle, Phys. Rev. Lett. 88, 172501 (2002)

[164] F. E. Close and A. W. Thomas, Phys. Lett. B212, 227 (1988)

[165] I. C. Cloët et al. Few-Body Systems 46, 1 (2009)

[166] K. Nagata and A. Hosaka, Mod. Phys. Lett. A23, 2397 (2008) 